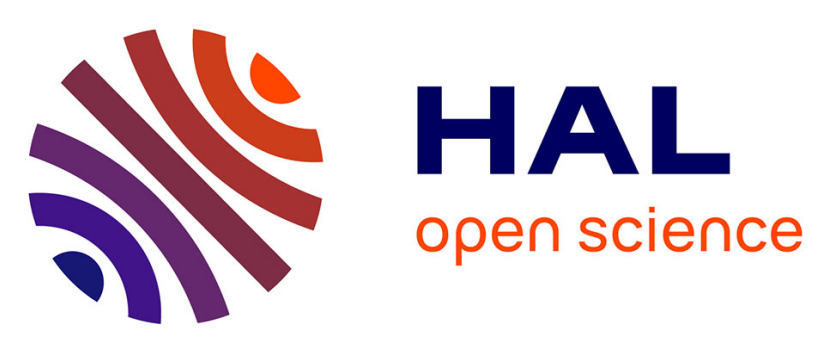

\title{
Fast Joint Multiband Reconstruction from Wideband Images Based on Low Rank Approximation
}

\author{
M. Amine Amine Hadj-Youcef, François Orieux, Alain Abergel, Aurélia \\ Fraysse
}

\section{- To cite this version:}

M. Amine Amine Hadj-Youcef, François Orieux, Alain Abergel, Aurélia Fraysse. Fast Joint Multiband Reconstruction from Wideband Images Based on Low Rank Approximation. IEEE Transactions on Computational Imaging, inPress, pp.1-1. 10.1109/TCI.2020.2998170 . hal-02778981

\section{HAL Id: hal-02778981 \\ https://hal.science/hal-02778981}

Submitted on 4 Jun 2020

HAL is a multi-disciplinary open access archive for the deposit and dissemination of scientific research documents, whether they are published or not. The documents may come from teaching and research institutions in France or abroad, or from public or private research centers.
L'archive ouverte pluridisciplinaire $\mathbf{H A L}$, est destinée au dépôt et à la diffusion de documents scientifiques de niveau recherche, publiés ou non, émanant des établissements d'enseignement et de recherche français ou étrangers, des laboratoires publics ou privés. 


\title{
Fast Joint Multiband Reconstruction from Wideband Images Based on Low-Rank Approximation
}

\author{
M. Amine Hadj-Youcef, Fraņcois Orieux, Alain Abergel, Aurélia Fraysse
}

\begin{abstract}
Multispectral imaging systems are increasingly used in many scientific fields. However multispectral images generally present spectral and spatial limitations: the spectral information within each band is lacking because of spectral integration over the band, and the spatial resolution is limited due to the spatial convolution by spectrally variant Point Spread Functions which introduce a spatial variant blur. To address the ill-posed inverse problem of reconstruction from wideband images, we propose a new approach combining a precise instrument model for the degraded multispectral images together with a spectral approximation on a low-rank subspace of the object. The reconstruction is based on the minimization of a convex objective function composed of a data fidelity and an edge-preserving regularization term. The proposed half-quadratic algorithm alternates between the minimization of a quadratic and a separable problem, and we show that both closed-form solutions are available and tractable. Therefore, even with a non-stationary data model, the algorithm is very fast and results are obtained in a few seconds.

Several tests are performed for multispectral data to be taken by MIRI, the mid-infrared imager of the future James Webb Space Telescope (JWST). The reconstruction results show a significant increase in spatial and spectral resolutions compared to state-of-the-art methods. Our proposed algorithm allows us to recover the spectroscopic information contained in the wideband multispectral images and to provide hyperspectral images with a homogenized spatial resolution over the entire spectral range.
\end{abstract}

Index Terms-Inverse Problems. Multispectral Imaging. Hyperspectral Imaging. Deconvolution. Image Reconstruction

\section{INTRODUCTION}

$\mathbf{M}$ ULTISPECTRAL imaging systems are used in many fields, e.g., astrophysics [1], remote sensing [2], medicine [3] or microscopy [4]. These imaging systems produce integrated multispectral images by observing a two spatial and one spectral dimensions, that is a $2 \mathrm{D}+\lambda$ object. Multispectral images have the benefits over hyperspectral images to have much larger field of view, better spatial resolution and higher sensitivity. However, they suffer from several undesirable spatial and spectral degradations. Firstly, spectral information is lacking because of the detector integration over wide spectral bands, resulting in an important subsampling. Secondly, the spatial resolution is limited by a $2 \mathrm{D}$ convolution of the $2 \mathrm{D}+\lambda$ object with the spectrally varying optical response or PSF (Point Spread Function). Generally, due to the diffraction theory [5], the longer the wavelength

M. Amine Hadj-Youcef, Francois Orieux and Aurélia Fraysse are with Laboratoire des Signaux et Systèmes (L2S), Université Paris-Saclay, CNRS, CentraleSupélec, 3 rue Joliot-Curie, 91192 Gif-sur-Yvette, France. e-mail: hadjyoucef . amine@gmail. fr

Alain Abergel is with the Institut d'Astrophysique Spatiale, Université Paris-Saclay, CNRS, 91405 Orsay, France.

Manuscript received September, 2019; Accepted May 18, 2020. the wider the PSF. Moreover, the spectral content of the input object depends on the pixel position in general. As a result, the blurring of multispectral images, which are integrated over a wide spectral range, is spatially varying. Furthermore the observations are made on wide bands, increasing the effect of spatial varying PSF.

The state-of-the-art approach generally neglects spectral and spatial variations of the PSF within a band [6] as well as correlations between bands, the reconstruction becoming an independent 2D deconvolution of each image [7]. In that case, the approach remains limited to systems with narrow spectral bands, which is generally not the case for multispectral imaging systems. Also, each spectral image is processed independently, and the spatial resolution is different for each processed image.

Other works focus on the variability of the PSF, especially for image deconvolution (e.g., [8], [9]) where the shiftvariant PSF is approximated with a linearly interpolated PSF. However, such a technique is generally not applicable to multispectral images since the spatial variations of the blurring are mainly due to the spatial variations of the spectral content of the object.

Another technique commonly used for astronomical images (e.g., [10], [11]) is the PSF homogenization between images obtained from different spectral bands or instruments. It consists of convolving images with appropriate kernels such that they appear as if they were measured with the same band or instrument. This approach is straightforward and simple, however, it introduces an additional blur and does not allow spectral reconstruction.

To address the problems of strong spectral subsampling and limitations of the spatial resolution due to the $2 \mathrm{D}$ convolution by a varying PSF, and to perform joint processing of all wide spectral bands, we propose to consider a linear spectral model together with a precise model of the multispectral instrument. Our goal is to derive an estimate of the $2 \mathrm{D}+\lambda$ object with the best spatial and spectral resolutions from the multispectral images. The proposed spectral modeling allows the description of the complexity of the input object spectra with a small number of components. This is common for hyperspectral images, in particular with a linear mixing model [12]-[14]. It was first proposed in [15] for the analysis of in-situ Mars surface images and, since then, has been used in several applications and methods [14], [16]-[19], also with spatial and spectral correlations and image enhancement methods based on total variation [20], [21]. In the context of multispectral imaging, the spectral modeling is less common except in pansharpening techniques [22]. It also is not adapted 
to spectrally varying PSF. Therefore we combine spectral modeling with an instrument model that correctly describes the spectral dependence of the PSF (and the detector sampling) to build a forward model of the data.

We show that all spectral and spatial information are embedded in the forward model, without any additional approximation. We also propose a fast convex reconstruction algorithm, where the two steps of the half-quadratic iterative algorithm are closed-form expression and tractable, for the joint processing of all wideband images. Our algorithm is very fast and can restore spatial details with identical resolution in all bands and better performances than the state-of-the-art TV-based image restoration. The proposed algorithm is tested for the reconstruction of three different hyperspectral objects that cover most of the encountered cases, including a simulated astrophysical object. The results clearly show a strong improvement in spectral and spatial resolutions compared to the state-of-the-art method [23]-[25]. The imaging system considered for this application is the Mid-InfraRed Instrument (MIRI) imager [26] on board the future James Webb Space Telescope (JWST) [27], which will be from 2021 the most ambitious telescope ever launched in space.

The paper is organized as follows. Section II presents the proposed methodology, first the instrument model developed for the imaging system (Section II-A), then the object representation model based on the linear mixing model (Section II-B) and finally the forward model (Section II-C). The reconstruction algorithm based on regularization methods is presented in Section III. Reconstruction results, including a comparison with a state-of-the-art method, and a discussion are presented in Section IV for the MIRI imager of the JWST. Finally, we conclude our work and provide perspectives in Section V.

\section{DATA MODEL}

\section{A. Instrument Model}

The hyperspectral object of interest is defined by

$$
\phi(x, y, \lambda): \mathbb{R}^{2} \times \mathbb{R}_{+} \rightarrow \mathbb{R}
$$

where $(x, y) \in \mathbb{R}^{2}$ represent the spatial dimensions and $\lambda \in$ $\mathbb{R}_{+}$represents the spectral one.

The imaging system consists of an optical system, a spectral filter, and a detector. Because of the optical diffraction theory, the optical system response is carried out by a 2D convolution [5] between the hyperspectral object $\phi$ and the spectralvariant PSF, $h$, assumed to be known, as $\iint_{\mathbb{R}^{2}} \phi\left(x^{\prime}, y^{\prime}, \lambda\right) h(x-$ $\left.x^{\prime}, y-y^{\prime}, \lambda\right) \mathrm{d} x^{\prime} \mathrm{d} y^{\prime}$. Hence, the final 2D images are impacted by a non-stationary spectrally integrated PSF that depends on the unknown spectral content of the object and also limits the spatial resolution of the images. Note that we assume that the monochromatic PSFs are spatially invariant. This may not be the case, but the amplitudes of the spatial variations of the monochromatic PSFs over the detector are generally lower than the amplitudes of the spectral variations of the PSF over the wide spectral bands of multispectral images.

For wideband imaging, the blurred object is spectrally filtered over $P$ wide spectral bands $\omega_{p}(\lambda), p=1, \ldots, P$, where $\omega_{p}$ is a spectral windowing, generally given by the product of the filter transmission and the detector quantum efficiency.

Then the object is integrated within each band and sampled pixel-by-pixel on the detector matrix, thus forming a discrete spectral image. Therefore, spectroscopic information of the hyperspectral object is reduced to only $P$ discrete values. This represents a severe degradation of the hyperspectral object since $P$ is usually a small number.

Spatial integration at pixel $(i, j)$ corresponds to multiplication by a square indicator function $b_{\mathrm{d}}$ on a two-dimensional sampling grid $\mathcal{G}_{\mathrm{d}}=\left\{i T_{x}, j T_{y}\right\}_{i ; j=1}^{N_{i} ; N_{j}}$ where $N_{i}, \quad N_{j}$ and $T_{x}, T_{y}$ are the number of pixels and the spatial sampling steps along $x$ and $y$, respectively. An additive term $n_{i, j}^{p}$ is added to the data to account for the detector noise. Finally, the complete equation of the imaging system model is given by

$$
\begin{gathered}
g_{i, j}^{p}=\iiint \iint \phi\left(x^{\prime}, y^{\prime}, \lambda\right) h\left(x-x^{\prime}, y-y^{\prime}, \lambda\right) \mathrm{d} x^{\prime} \mathrm{d} y^{\prime} \\
\omega_{p}(\lambda) b_{\mathrm{d}}\left(x-i T_{x}, y-j T_{y}\right) \mathrm{d} x \mathrm{~d} y \mathrm{~d} \lambda+n_{i, j}^{p} .
\end{gathered}
$$

The model in (1) establishes a relation between the continuous hyperspectral object $\phi$ and the discrete images $g^{p}, p=$ $1, \ldots, P$ through the instrument response. It includes a spectral windowing and five integration for spatial convolution and spatio-spectral sampling.

\section{B. Object Model}

The object model is critical since the spectral information is seriously lacking in multispectral images. However it is important to note that the spatial structure of multispectral images depends on the spectral content of the object $\phi$ through the spectral dependence of the PSF $h$. In a previous work, [28] we proposed a spline model for spectral distribution and introduced strong smoothing prior in order to overcome the spectral subsampling, without the possibility of accurate spectral retrieval.

Instead, in order to overcome the lack of spectral information in the data, we propose here to model the hyperspectral object with a low rank approximation thanks to the linear mixing model of [15] considering a small number of components while keeping high spectral resolution. Therefore, the object is represented by a sum of $M$ high-resolution known spectral components, $s^{m}(\lambda), m=1, \ldots, M$, weighted by $M$ mixing coefficients $f_{k, l}^{m}$ associated with each spatial position $(k, l)$. Hence the object is decomposed, thanks to an indicator

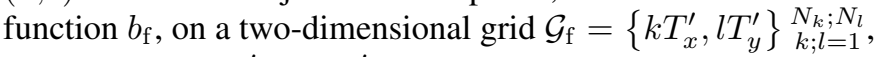
where $N_{k}, N_{l}, T_{x}^{\prime}$ and $T_{y}^{\prime}$ are the number of samples and the sampling steps according to dimensions $x$ and $y$, respectively. This yields

$$
\phi(x, y, \lambda)=\sum_{m, k, l=1}^{M, N_{k}, N_{l}} f_{k, l}^{m} b_{\mathrm{f}}\left(x-k T_{x}^{\prime}, y-l T_{y}^{\prime}\right) s^{m}(\lambda) .
$$

Since only multispectral data are available, the spectral components $s^{m}(\lambda)$ are supposed known, because there is not enough data to estimate them jointly. However, they can be extracted 
from previous hyperspectral measurements of similar objects with a principal component analysis (PCA) [29], non-negative matrix factorization (NMF) [30], or by elements of a given dictionary if, for instance, a material is known to be present, which is often the case. Nonetheless, we suppose that no specific meaning is attached to $s_{m}$ except for their ability to represent the spectral variability that lives in a low rank space.

\section{Forward Model}

The forward model is obtained by substituting (2) in (1) yielding the linear model

$$
g_{i, j}^{p}=\sum_{m=1}^{M} \sum_{k=1}^{N_{k}} \sum_{l=1}^{N_{l}} H_{i ; j, k ; l}^{p, m} f_{k, l}^{m}+n_{i, j}^{p}
$$

with

$$
\begin{aligned}
& H_{i ; j, k ; l}^{p, m}=\int \omega_{p}(\lambda) s^{m}(\lambda) \iint\left[h\left(x^{\prime}, y^{\prime}, \lambda\right)_{x^{\prime}, y^{\prime}}^{*}\right. \\
& \left.b_{\mathrm{f}}\left(x^{\prime}-k T_{x}^{\prime}, y^{\prime}-l T_{y}^{\prime}\right)\right] b_{\mathrm{d}}\left(x-i T_{x}, y-j T_{y}\right) \mathrm{d} x \mathrm{~d} y \mathrm{~d} \lambda,
\end{aligned}
$$

where $\underset{x^{\prime}, y^{\prime}}{*}$ indicates the $2 \mathrm{D}$ convolution. Combining integrals leads to

$$
H_{i j k l}^{p m}=\int \omega_{p}(\lambda) s^{m}(\lambda) \bar{h}\left(k T_{x}^{\prime}-i T_{x}, l T_{x}^{\prime}-j T_{x}, \lambda\right) \mathrm{d} \lambda
$$

with

$$
\begin{gathered}
\bar{h}\left(k T_{x}^{\prime}-i T_{x}, l T_{x}^{\prime}-j T_{x}, \lambda\right)=\iint h\left(x^{\prime}, y^{\prime}, \lambda\right)_{x^{\prime}, y^{\prime}}^{*} \\
b_{\mathrm{f}}\left(x^{\prime}-k T_{x}^{\prime}, y^{\prime}-l T_{y}^{\prime}\right) b_{\mathrm{d}}\left(x-i T_{x}, y-j T_{y}\right) \mathrm{d} x \mathrm{~d} y,
\end{gathered}
$$

that is a spatial super-resolution model since this model computes the exact contribution of each pixel on each detector element. However, the computation of each $H_{i j k l}^{p m}$ element can be computationally heavy. Nevertheless, taking $T_{x}^{\prime}=T_{x}$ and $T_{y}^{\prime}=T_{y}$ simplify Eq. (5) as

$$
\begin{aligned}
H_{i j k l}^{p m} & =\int \omega_{p}(\lambda) s^{m}(\lambda) \bar{h}\left((k-i) T_{x},(l-j) T_{x}, \lambda\right) \mathrm{d} \lambda \\
& =H_{k-i, l-j}^{p m}
\end{aligned}
$$

and in that case, the linear model becomes a numerical spatial convolution.

By denoting $\boldsymbol{g}^{p}$ the vector of data in the band $p$, the forward model can be written as

$$
\boldsymbol{g}^{p}=\sum_{m=1}^{M} \boldsymbol{H}^{p, m} \boldsymbol{f}^{m}+\boldsymbol{n}^{p}, \quad p=1,2, \ldots, P,
$$

where the $p$-th image $\boldsymbol{g}^{p} \in \mathbb{R}^{N_{i} N_{j}}$ is a sum of $M$ discrete spatial convolutions of mixing coefficients $\boldsymbol{f}^{m} \in \mathbb{R}^{N_{k} N_{l}}, m=$ $1, \ldots, M$, with convolution matrix $\boldsymbol{H}^{p, m} \in \mathbb{R}^{N_{i} N_{j} \times N_{k} N_{l}}$, plus an additive noise $\boldsymbol{n}^{p} \in \mathbb{R}^{N_{i} N_{j}}$. The convolution matrix $\boldsymbol{H}^{p, m}$ models the spatial impact of the spectral distribution $m$ on the $p$-th image, describing the spatial variation of the response.

We propose to process data from all the bands together in order to reconstruct the total spectral information, instead of a band per band separated processing [25], [31]. This has the advantage of taking into account correlations between bands. Therefore, by concatenating all images in one vector, we obtain the following multi-observations forward model

$$
\boldsymbol{g}=\boldsymbol{H} \boldsymbol{f}+\boldsymbol{n},
$$

where $\boldsymbol{g}^{T}=\left[\boldsymbol{g}^{1}, \ldots, \boldsymbol{g}^{P}\right]^{T}, \boldsymbol{f}^{T}=\left[\boldsymbol{f}^{1}, \ldots, \boldsymbol{f}^{M}\right]^{T}$, and $\boldsymbol{n}^{T}=$ $\left[\boldsymbol{n}^{1}, \ldots, \boldsymbol{n}^{P}\right]^{T}$.

The full system observation matrix

$$
\boldsymbol{H}=\left[\begin{array}{ccc}
\boldsymbol{H}^{1,1} & \cdots & \boldsymbol{H}^{1, M} \\
\vdots & \ddots & \vdots \\
\boldsymbol{H}^{P, 1} & \cdots & \boldsymbol{H}^{P, M}
\end{array}\right]
$$

is a non-square non-Tœplitz matrix with Tœplitz block components $\boldsymbol{H}^{p, m}$ representing the contribution of templates $m$ to image $p$. For computational efficiency, the convolutions are done in the Fourier domain [32].

\section{RECONSTRUCTION ALGORITHM}

\section{A. Variational formulation}

The problem of reconstructing $f$ defined in Eq. (9) is illposed because of the convolution, leading to noise amplification. The common approach in this case is to add prior information about the solution, as in the regularized least square method [33]. Therefore, the solution $\widehat{f}$ is obtained as a minimizer of an objective function $\mathcal{J}(\boldsymbol{f})$

$$
\widehat{\boldsymbol{f}}=\underset{\boldsymbol{f}}{\operatorname{argmin}}\{\mathcal{J}(\boldsymbol{f})=\mathcal{Q}(\boldsymbol{f}, \boldsymbol{g})+\mu \mathcal{R}(\boldsymbol{f})\},
$$

where $\mathcal{Q}(\boldsymbol{f}, \boldsymbol{g})$ is a data fidelity term, $\mathcal{R}(\boldsymbol{f})$ a regularization term added to correct the ill-conditioning of the problem and $\mu \geq 0$ a regularization parameter to tune the trade-off between both these terms.

The noise is modeled by an identically independent Gaussian distribution for the sake of clarity, leading to $\mathcal{Q}(\boldsymbol{f}, \boldsymbol{g})=$ $\|\boldsymbol{g}-\boldsymbol{H} \boldsymbol{f}\|_{2}^{2}$. The proposed algorithm can be extended to circulant spatially correlated Gaussian noise without restriction. Concerning the regularization term, many possibilities have been explored in the literature. For instance, one can consider Tikhonov regularization [34], total variation [35], wavelet-domain regularization [36], [37], or half-quadratic regularization [38], [39]. Here we are interested in the reconstruction of a spatially smooth hyperspectral object with sharp edges. The prior information is then introduced by penalizing the horizontal and vertical differences between neighboring pixels of each mixing coefficient. In that case, a multichannel regularization term is defined as

$$
\mathcal{R}(\boldsymbol{f})=\sum_{m, k, l=1}^{M, N_{k}, N_{l}} \varphi \underbrace{\left(\boldsymbol{f}_{k+1, l}^{m}-\boldsymbol{f}_{k, l}^{m}\right)}_{\left[\boldsymbol{D}_{v} \boldsymbol{f}^{m}\right]_{k, l}}+\varphi \underbrace{\left(\boldsymbol{f}_{k, l+1}^{m}-\boldsymbol{f}_{k, l}^{m}\right)}_{\left[\boldsymbol{D}_{h} \boldsymbol{f}^{m}\right]_{k, l}},
$$

where $\varphi$ is the penalty function. $\boldsymbol{D}_{h}$ and $\boldsymbol{D}_{v}$ are firstorder finite difference operators, with circularity conditions $f_{N_{k}+1, l}^{m}=f_{1, l}^{m}$ and $f_{k, N_{l}+1}^{m}=f_{k, 1}^{m}$. A classical choice is the quadratic function $\varphi(x)=x^{2}$ that gives a differentiable 
objective function and an explicit solution. However, spatial sharp edges are smoothed.

To overcome this limitation we propose to use a nonquadratic penalty function. Several methods are found in the literature such as methods based on partial differential equation [40], total variation (TV) $\left(\ell_{1}\right.$-norm of the gradient) [35], [41] half-quadratic regularization $\left(\ell_{2} \ell_{1}\right.$-norm) [38], [39], [42] or majoration-minimization algorithm [43]. We use the method developed in [39] for several reasons. Firstly, the minimization of the objective function is done through alternating quadratic and separable minimization problems. Secondly, the quadratic solution is directly tractable thanks to the invertibility of the Hessian matrix, leading to a very fast algorithm. Thirdly, a variety of penalty functions can be used, such as the convex Huber function

$$
\varphi(x)= \begin{cases}x^{2}, & \text { if }|x|<s, s \in \mathbb{R} \\ 2 s|x|-s^{2}, & \text { otherwise, }\end{cases}
$$

which is used in the rest of this work to prevents the cartoonlike effect given by TV penalization. The parameter $s>0$ is a threshold parameter that defines the transition from a quadratic to a linear penalization. The half-quadratic regularization proposed by Geman \& Yang in [39] consists of introducing $N_{k} \times N_{l}$ horizontal and vertical auxiliary variables, $b$, such that the penalty function $\varphi$ is expressed as the minimum wrt. $b$ of the sum of a quadratic function $(x-b)^{2} / 2$ and an auxiliary function $\xi(b)$ (that depends on $\varphi$ )

$$
\varphi(x)=\inf _{b} \psi(b)=\inf _{b} \frac{1}{2}(x-b)^{2}+\xi(b), \quad \forall x \in \mathbb{R} .
$$

The construction relies on convex duality, with $\xi(b)=2 s|b|$ for Huber potential, as described in Appendix B. The practical role of these auxiliary variables $b$ can be seen as shifting the quadratic function to a suitable position such as the cost at high gradient values is lower compared to the cost of the quadratic regularization. The auxiliary function relies on the convex duality and Legendre-Fenchel transform [38], [39], [42], [44]. Consequently, an augmented objective function $\mathcal{J}^{*}$ is defined such that

$$
\inf _{\boldsymbol{b}_{h}, \boldsymbol{b}_{v}} \mathcal{J}^{*}\left(\boldsymbol{f}, \boldsymbol{b}_{h}, \boldsymbol{b}_{v}\right)=\mathcal{J}(\boldsymbol{f}) .
$$

Here $\boldsymbol{b}_{h}$ and $\boldsymbol{b}_{v}$ are vector representations of the stack of auxiliary variables along the horizontal and vertical directions. Therefore, the multichannel half-quadratic solution is obtained by minimizing the augmented objective function

$$
\left(\widehat{\boldsymbol{f}}, \widehat{\boldsymbol{b}}_{h}, \widehat{\boldsymbol{b}}_{v}\right)=\underset{\boldsymbol{f}, \boldsymbol{b}_{h}, \boldsymbol{b}_{v}}{\operatorname{argmin}} \mathcal{J}^{*}\left(\boldsymbol{f}, \boldsymbol{b}_{h}, \boldsymbol{b}_{v}\right) .
$$

Since the criterion is globally convex [44], the computation of the joint minimizer of $\mathcal{J}^{*}\left(\boldsymbol{f}, \boldsymbol{b}_{h}, \boldsymbol{b}_{v}\right)$ with respect to $\left(\boldsymbol{f}, \boldsymbol{b}_{h}, \boldsymbol{b}_{v}\right)$ is achieved by iterating the following two-stage process until convergence

$$
\left\{\begin{array}{l}
\widehat{\boldsymbol{f}}^{(k)}=\underset{\boldsymbol{f}}{\operatorname{argmin}} \mathcal{J}^{*}\left(\boldsymbol{f}, \boldsymbol{b}_{h}^{(k-1)}, \boldsymbol{b}_{v}^{(k-1)}\right), \\
\widehat{\boldsymbol{b}}_{h}^{(k)}, \widehat{\boldsymbol{b}}_{v}^{(k)}=\underset{\boldsymbol{b}_{h}, \boldsymbol{b}_{v}}{\operatorname{argmin}} \mathcal{J}^{*}\left(\boldsymbol{f}^{(k)}, \boldsymbol{b}_{h}, \boldsymbol{b}_{v}\right) .
\end{array}\right.
$$

\section{B. Fast mixing coefficients $\widehat{\boldsymbol{f}}$ update}

From (16) we have the quadratic criterion

$$
\begin{aligned}
& \widehat{\boldsymbol{f}}=\underset{\boldsymbol{f}}{\operatorname{argmin}}\left\{\|\boldsymbol{g}-\boldsymbol{H} \boldsymbol{f}\|_{2}^{2}+\right. \\
&\left.\mu\left(\left\|\overline{\boldsymbol{D}}_{h} \boldsymbol{f}-\boldsymbol{b}_{h}\right\|_{2}^{2}+\left\|\overline{\boldsymbol{D}}_{v} \boldsymbol{f}-\boldsymbol{b}_{v}\right\|_{2}^{2}\right)\right\}
\end{aligned}
$$

with $\overline{\boldsymbol{D}}_{h}=\operatorname{diag}\left\{\boldsymbol{D}_{h}, \ldots, \boldsymbol{D}_{h}\right\}$. and $\overline{\boldsymbol{D}}_{v}=$ $\operatorname{diag}\left\{\boldsymbol{D}_{v}, \ldots, \boldsymbol{D}_{v}\right\}$, two diagonal-block matrices. The minimizer is explicit and is obtained by canceling the gradient. This yields

$$
\begin{aligned}
\widehat{\boldsymbol{f}}=\underbrace{\left(\boldsymbol{H}^{T} \boldsymbol{H}+\mu\left(\overline{\boldsymbol{D}}_{h}^{T} \overline{\boldsymbol{D}}_{h}+\overline{\boldsymbol{D}}_{v}^{T} \overline{\boldsymbol{D}}_{v}\right)\right)}_{\boldsymbol{Q}}-1 & \underbrace{\left(\boldsymbol{H}^{T} \boldsymbol{g}+\mu\left(\overline{\boldsymbol{D}}_{h}^{T} \boldsymbol{b}_{h}+\overline{\boldsymbol{D}}_{v}^{T} \boldsymbol{b}_{v}\right)\right)}_{\boldsymbol{q}},
\end{aligned}
$$

where the Hessian matrix $\boldsymbol{Q} \in \mathbb{R}^{M N_{k} N_{l} \times M N_{k} N_{l}}$ is a blockcirculant matrix, and $\boldsymbol{q} \in \mathbb{R}^{M N_{k} N_{l}}$ is a multichannel vector.

A common computational approach of $\widehat{f}$ in Eq. (19) relies on solving the linear system $\boldsymbol{Q} \boldsymbol{f}=\boldsymbol{q}$ without requiring the inversion of $\boldsymbol{Q}$ thanks to iterative algorithms, e.g., conjugate gradient. On the contrary, in this paper the closed form solution is computed thanks to a fast and exact inversion of $Q$ (see Appendix A for details).

\section{Auxiliary variables $\widehat{\boldsymbol{b}}_{h}, \widehat{\boldsymbol{b}}_{v}$ update}

From (17) we have

$$
\widehat{\boldsymbol{b}}_{h}, \widehat{\boldsymbol{b}}_{v}=\underset{\boldsymbol{b}_{h}, \boldsymbol{b}_{v}}{\operatorname{argmin}} \sum_{m, k, l=1}^{M, N_{k}, N_{l}} \psi\left(\left[\boldsymbol{b}_{h}\right]_{k, l}\right)+\psi\left(\left[\boldsymbol{b}_{v}\right]_{k, l}\right)
$$

where $\psi$ is a convex and differentiable function defined in Appendix B. Moreover, the update equation for each auxiliary variable is explicit and separable with

$$
\left[\widehat{\boldsymbol{b}}_{*}^{m}\right]_{k, l}=\underset{\left[\boldsymbol{b}_{*}^{m}\right]_{k, l}}{\operatorname{argmin}} \psi\left(\left[\boldsymbol{b}_{*}^{m}\right]_{k, l}\right) .
$$

The computation of the minimizers in (21) is straightforward and it is detailed in Appendix B. Finally, we obtain

$$
\widehat{\boldsymbol{b}}_{*}=\overline{\boldsymbol{D}}_{*} \boldsymbol{f}-\frac{1}{2} \varphi^{\prime}\left(\overline{\boldsymbol{D}}_{*} \boldsymbol{f}\right)
$$

where $\varphi^{\prime}$ is the first derivative of the Huber function given by

$$
\varphi^{\prime}(x)= \begin{cases}2 x, & \text { if }|x|<s, \\ 2 s \operatorname{sign}(x), & \text { otherwise. }\end{cases}
$$

The proposed Fast Joint Multiband Reconstruction (FJMR) algorithm is summarized in a pseudo-algorithm form in Algorithm 1.

\section{EXPERIMENTAL RESULTS}

In this section we present tests and comparisons of the proposed algorithm for the reconstruction of three hyperspectral objects having different spatial and spectral distributions. One 


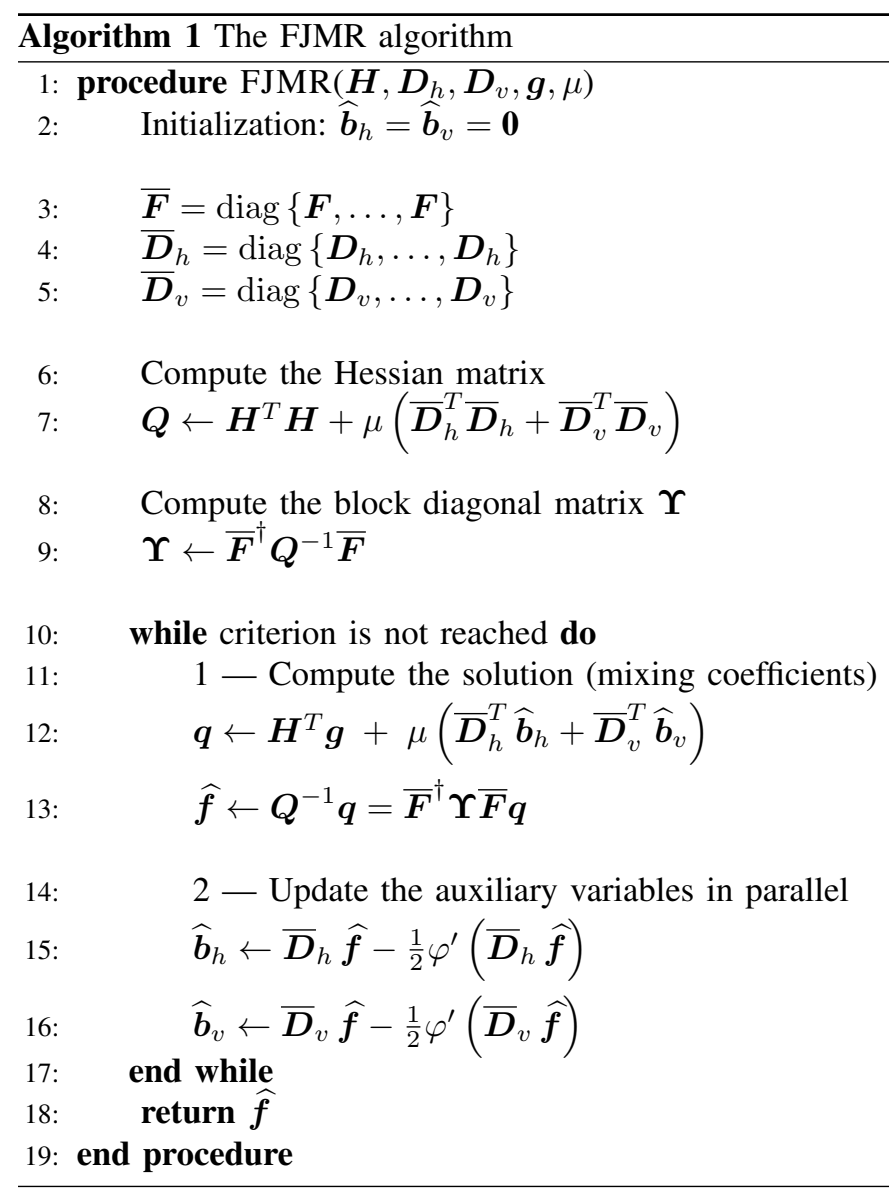

is the model of an astrophysical object and the other ones are synthetic objects. The multispectral imaging system we are considering is the MIRI imager [26] on board the JWST [27]. Note that we suppose the common case where the spectral components $s^{m}$ are known and extracted from hyperspectral measurements or within a dictionary. We compare our results to the state-of-the-art TV deconvolution implemented with the primal dual Chambolle-Pock algorithm [25] that minimizes

$$
\hat{\boldsymbol{f}}^{p}=\underset{\boldsymbol{f}^{p}}{\operatorname{argmin}}\left\|\boldsymbol{g}^{p}-\boldsymbol{H}^{p} \boldsymbol{f}^{p}\right\|_{2}^{2}+\mu\left\|\nabla \boldsymbol{f}^{p}\right\|_{1}
$$

for each band $p$, with $\nabla \boldsymbol{f}^{p}$ the spatial gradient of the image band $p$. Here $\boldsymbol{H}^{p}$ is the PSF integrated over the spectral band $p$ assuming a flat spectrum. To complement the results we compare also our algorithm to the $l_{2}$ reconstruction defined as

$$
\hat{\boldsymbol{f}}^{p}=\underset{\boldsymbol{f}^{p}}{\operatorname{argmin}}\left\|\boldsymbol{g}^{p}-\boldsymbol{H}^{p} \boldsymbol{f}^{p}\right\|_{2}^{2}+\mu\left\|\nabla \boldsymbol{f}^{p}\right\|_{2}^{2}
$$

that is the classical regularized least-squares method, with a conjugate-gradient as optimization algorithm.

The hyperparameter $\mu$ is hand tuned in order to minimize the $\ell_{2}$ reconstruction error. The algorithms are coded using Python 2.7 and executed on a laptop machine with $16 \mathrm{~GB}$ of RAM and a processor Intel Core i7 CPU working at $2.50 \mathrm{GHz}$.

\section{A. The MIRI Imager of the JWST}

The optical system of the JWST is equipped with a 6.5 meters primary mirror composed of 18-hexagonal seg- ments. The analytic expression of the monochromatic PSF at one wavelength can theoretically be obtained by computing the Fourier transform of the transmittance of the telescope aperture, in accordance with the diffraction theory [5]. However, it is also necessary to take into account misalignments of the 18 segments and the optical path differences. Therefore the monochromatic PSFs are computed with WebbPSF [45], [46], the official PSF simulator for the JWST developed by the Space Telescope Science Institute (STScI) ${ }^{1}$. No analytical formula of the PSF $h$ is available, therefore all calculations to derive the convolution matrix $\boldsymbol{H}^{p, m}$ (Eq. (7)) are done numerically. Fig. 1 displays a few monochromatic PSFs computed at 6,12 , and $18 \mu \mathrm{m}$. We clearly see the spectral dependence of the PSF, i.e., the longer the wavelength the wider the PSF as expected from the diffraction theory.

The multispectral images are integrated over $P=9$ spectral widebands covering a spectral range from 5 to $30 \mu \mathrm{m}$ as shown in Fig. 2. The MIRI imager provides images with a field of view (FOV) of $74 \times 113$ arcsecond $^{2}$ using a unique infrared detector with a pixel scale of 0.11 arcsecond, i.e., a FOV per pixel of $0.11 \times 0.11$ arcsecond $^{2}$. Spatial variations of the PSF across the imager FOV have been measured on the flight model of the MIRI imager [6] with a width variation of the PSF across the field of view inferior to $5 \%$. Thus the hypothesis of spatially invariant monochromatic PSFs is justified.

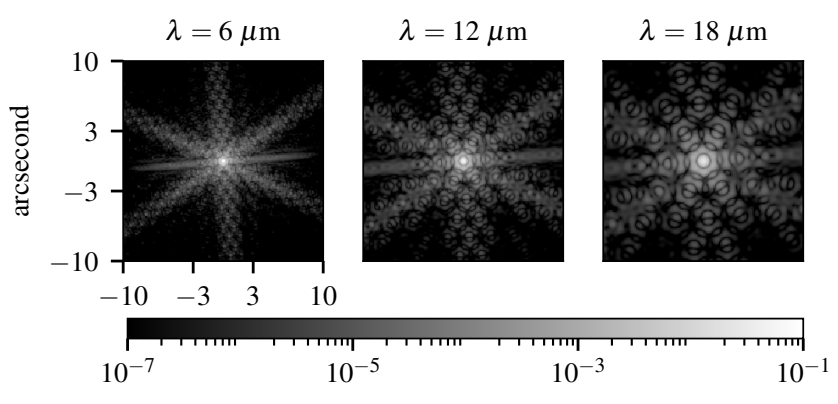

Fig. 1. Monochromatic PSF of the JWST/MIRI imager simulated at 6, 12, and $18 \mu \mathrm{m}$ using WebbPSF [45] and displayed in logarithmic scale.

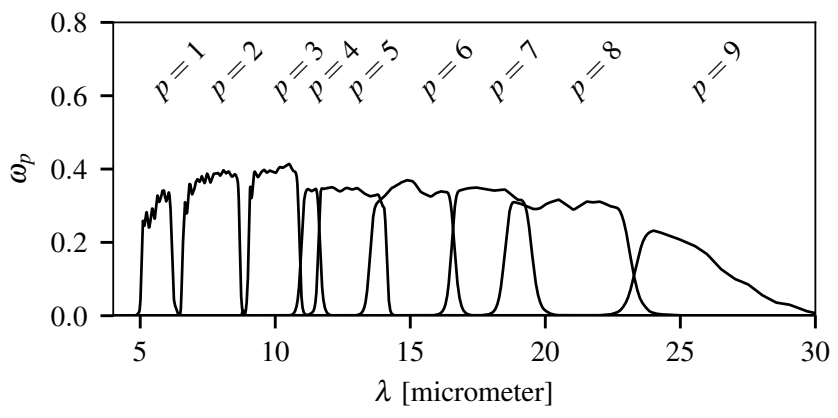

Fig. 2. Wide spectral bands $\omega_{p}$ of the JWST/MIRI Imager [47], from [48].

\footnotetext{
${ }^{1}$ http://www.stsci.edu/
} 


\section{B. Description of the hyperspectral objects}

Our algorithm has been tested using three hyperspectral objects which cover a significant range of spatial and spectral structures expected for astrophysical observations.

The first one, denoted by $\mathrm{Obj}_{1}$, is an astrophysical simulation of the HorseHead nebula [49], modeling a cloud of matter (dust and gas) illuminated by a bright $s^{2}{ }^{2}$, with $N_{k}=N_{l}=256$ spatial samples, and $N_{\lambda}=1000$ spectral samples uniformly distributed from 1 to $30 \mu \mathrm{m}$. Therefore, $\mathrm{Obj}_{1}$ is a full $2 \mathrm{D}+\lambda$ cube that is not constructed with the object model in Eq. (2), and original coefficients are not available. For the reconstruction, $M$ spectral components $s^{m}$ are extracted with a PCA [29]. For that object only $M=3$ components (shown in Fig. 3) are sufficient to explain $99.99 \%$ of the variance of the spectra. Note that other extraction techniques could be used such as the NMF [30], or blind source separations [18], but this is not necessary in our case.

Two hyperspectral objects with more complex spatial and spectral distributions, $\mathrm{Obj}_{2}$ and $\mathrm{Obj}_{3}$, are synthesized. Fig. 4 and Fig. 5 display the spectral components and the original mixing coefficients used to synthesize $\mathrm{Obj}_{2}$ and $\mathrm{Obj}_{3}$, respectively. For both objects, the spectral components are taken from [18]. They have been computed from real data obtained by the spectrometer of the Spitzer Space Telescope [50] which covers the same spectral range as the MIRI imager. For the mixing coefficients of $\mathrm{Obj}_{2}$ we take $M=3$ rectangular patterns of different size with sharp edges, each associated to one of the three spectral components. For $\mathrm{Obj}_{3}$ we take $M=2$ mixing coefficients in order to create a complex highfrequency spatial structure with a smooth horizontal gradient.

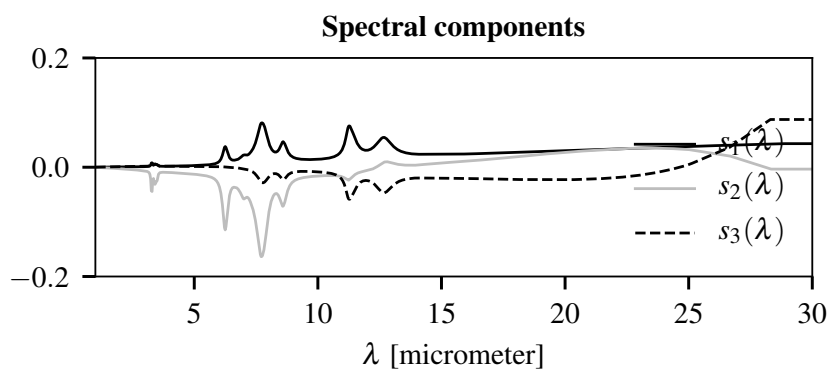

Fig. 3. Three spectral components extracted from $\mathrm{Obj}_{1}$ with a PCA. The third component is negative due to the PCA formalism. The curves are in input sky unit.

\section{Simulation of the multispectral data}

The $P=9$ images for $\mathrm{Obj}_{1}, \mathrm{Obj}_{2}$, and $\mathrm{Obj}_{3}$ are simulated using the MIRI imager instrument model in (1), and not the forward model with the mixing model in (9). We degrade the images with an additive zero-mean, white, Gaussian noise of different levels of Signal-to-Noise Ratio (SNR), that is 5, 10, 20, 30, and $40 \mathrm{~dB}$, defined as SNR $=10 \log _{10}\left(\|\boldsymbol{g}\|_{2}^{2} / N \sigma_{n}^{2}\right)$, where $\sigma_{n}$ is the standard deviation of the noise, and $N$ is the total number of pixels in $\boldsymbol{g}$.

\footnotetext{
${ }^{2}$ This $2 \mathrm{D}+\lambda$ object has been computed using state-of-the-art interstellar dust models and radiative transfer codes.
}

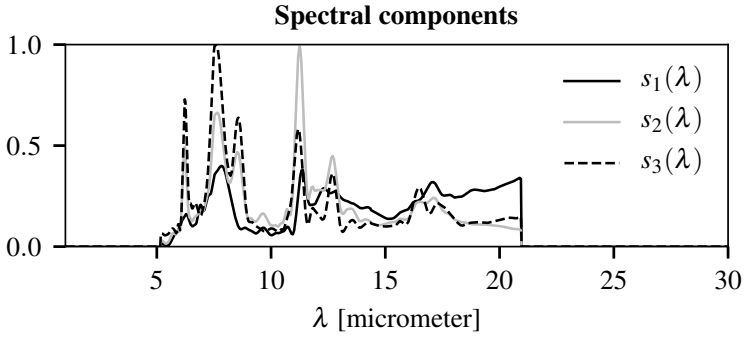

Original mixing coefficients

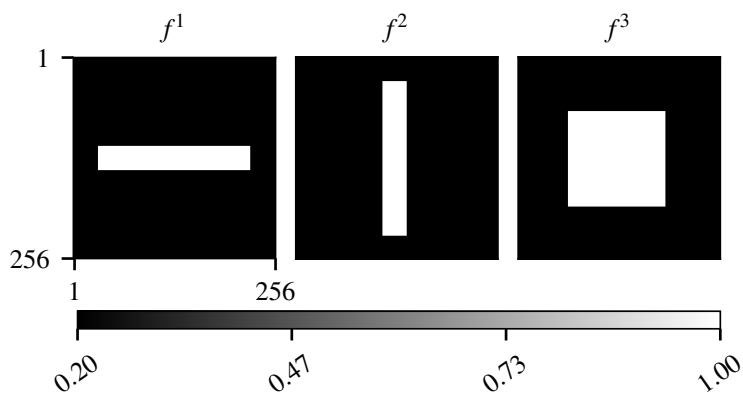

Fig. 4. Spectral components and mixing coefficients for $\mathrm{Obj}_{2}$. The curves are in input sky unit.
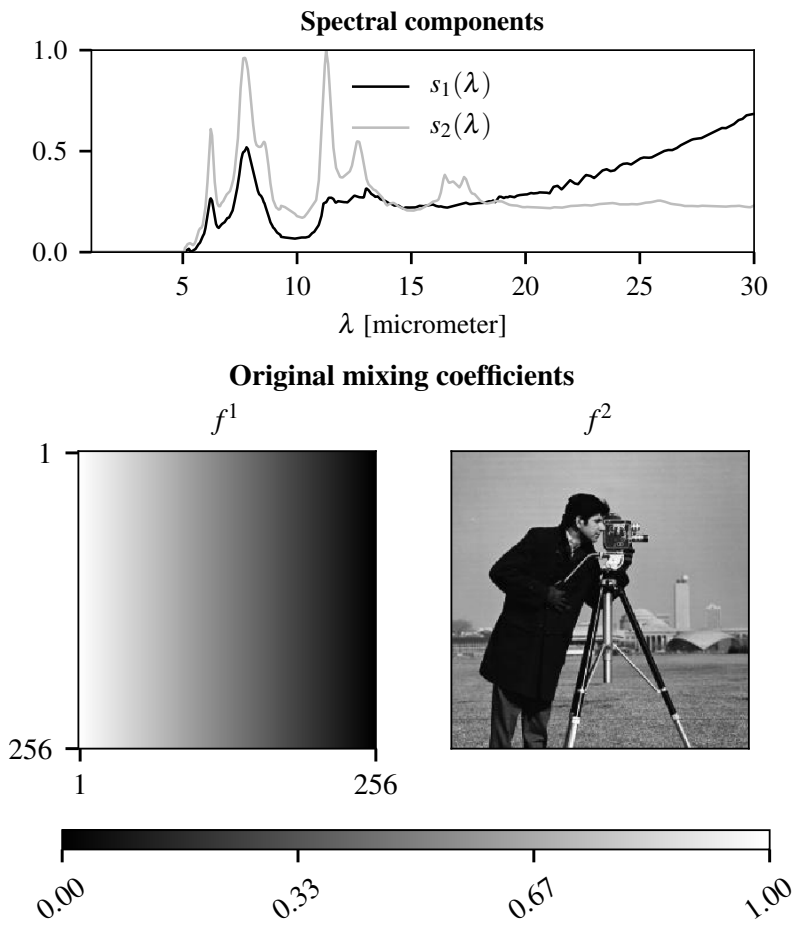

Fig. 5. Spectral components and mixing coefficients for $\mathrm{Obj}_{3}$. The curves are in input sky unit.

The simulated multispectral images with SNR $=30 \mathrm{~dB}$ and $p=1,4,7$ are displayed in the first row of Figs. 8, 9, and 10, for $\mathrm{Obj}_{1}, \mathrm{Obj}_{2}$ and $\mathrm{Obj}_{3}$, respectively. As expected, the blur increases for increasing wavelengths due to the convolution by a wavelength-variable PSF. The images have different intensities due to the spectral distribution integrated within 
TABLE I

RECONSTRUCTION ERRORS WITH 50 ITERATIONS.

\begin{tabular}{ccccc}
\hline Object & Error [\%] & Runtime [s] & $\mu$ & $s$ \\
\hline$\widehat{\mathrm{Obj}}_{1}$ & 0.67 & 4.34 & $5.99 \times 10^{-2}$ & 1.17 \\
$\widehat{\mathrm{Obj}}_{2}$ & 1.91 & 4.26 & $4.64 \times 10^{-2}$ & 0.03 \\
$\widehat{\mathrm{Obj}}_{3}$ & 4.91 & 3.08 & $5.99 \times 10^{-2}$ & 0.01 \\
\hline
\end{tabular}

the spectral bands and the width of each band. In any case these images are spatially degraded and contain poor spectral information.

\section{Estimation results for $\widehat{\boldsymbol{f}}, \widehat{\boldsymbol{b}}_{h}$ and $\widehat{\boldsymbol{b}}_{v}$}

For the reconstruction, the parameters $\mu$ and $s$ are chosen in order to minimize the reconstruction error (in \%) on sampled full $2 \mathrm{D}+\lambda$ cube (not coefficients)

$$
\operatorname{Error}(\mu, s)=\left\|\phi_{\text {orig }}-\phi_{\boldsymbol{f}}(\mu, s)\right\|_{2} /\left\|\phi_{\text {orig }}\right\|_{2} \times 100,
$$

with values reported in Table I. The spatial distributions of the estimated mixing coefficients $\widehat{f}$ are shown in Fig. 6 for $\mathrm{Obj}_{1}$ (top row), $\mathrm{Obj}_{2}$ (middle row) and $\mathrm{Obj}_{3}$ (bottom row). For $\mathrm{Obj}_{1}$ we see that $\widehat{\boldsymbol{f}}^{1}$ has a higher intensity than $\widehat{\boldsymbol{f}}^{2}$ and $\widehat{\boldsymbol{f}}^{3}$, due to the domination of the first spectral component in the spectral distribution of $\mathrm{Obj}_{1}$. For $\mathrm{Obj}_{2}$ and $\mathrm{Obj}_{3}$, the mixing coefficients appear properly unmixed and deconvolved.

The spatial distributions of the estimated auxiliary variables $\left(\widehat{\boldsymbol{b}}_{h}, \widehat{\boldsymbol{b}}_{v}\right)$, shown in Fig. 7 for $\mathrm{Obj}_{2}$ mimic the contours of the mixing coefficients, as expected from the half-quadratic minimization.

\section{E. Hyperspectral Reconstruction Results}

The reconstructed objects are computed using the linear mixing model in (2) and using the estimated mixing coefficients presented above. In Table I we present the reconstruction errors for the three objects.

All reconstruction errors are below 5\%. The smallest error $(0.67 \%)$ is obtained for $\widehat{\mathrm{Obj}}_{1}$ which contains neither sharp edge nor complex spectral features. For $\widehat{\mathrm{Obj}}_{2}$ and $\widehat{\mathrm{Obj}}_{3}$, reconstruction errors are $1.91 \%$ and $4.91 \%$ respectively, because of their more complex spatial and spectral contents.

For a better illustration of our reconstruction results and a comparison to results obtained using the state-of-the-art TV deconvolution algorithm, we discuss separately the spatial distribution and the spectral distribution obtained for each object.

a) Spatial distribution: The spatial distributions are illustrated in Figs. 8, 9, and 10 by taking three monochromatic images at wavelengths $\lambda=6,12,18 \mu \mathrm{m}$, belonging to the three spectral bands $p=1,4$ and 7, respectively. Figs. 9 and 10 are displayed with a spatial zoom to highlight details.

The proposed reconstructions show good performance. The dynamic range and the spatial distribution of the monochromatic images are well reconstructed with errors around $0.62 \%$ for $\mathrm{Obj}_{1}, 2.20 \%$ for $\mathrm{Obj}_{2}$, and $5.75 \%$ for $\mathrm{Obj}_{3}$. This illustrates the efficiency of the proposed algorithm for reconstruction at all wavelengths. The comparison between the first three lines of Figs. 9 and 10 illustrates the striking improvement of the spatial resolution. Our algorithm correctly recovers the sharp edges and small-scale gradients contained in the original objects. Thanks to the mixing model, the spatial resolution at all wavelengths is determined by the spatial resolution of the estimated mixing coefficients. Therefore, the reconstructed monochromatic images do not show any increasing blur with increasing wavelengths, unlike the input images and the images computed with the TV deconvolution. Moreover, TV deconvolution (which takes between 70 and 100 seconds for one image and 500 iterations) can only be done image by image and therefore cannot restore spatial details at small scales and at long wavelengths. In addition, the color bars show that the dynamic range is not properly restored with TV deconvolution. This is due to the integration over the wide spectral bands, and to the spectral dependence of the PSF within each wideband image which is neglected. The $l_{2}$ approach suffer the same problems that TV deconvolution with lesser quality results, as expected for this method.
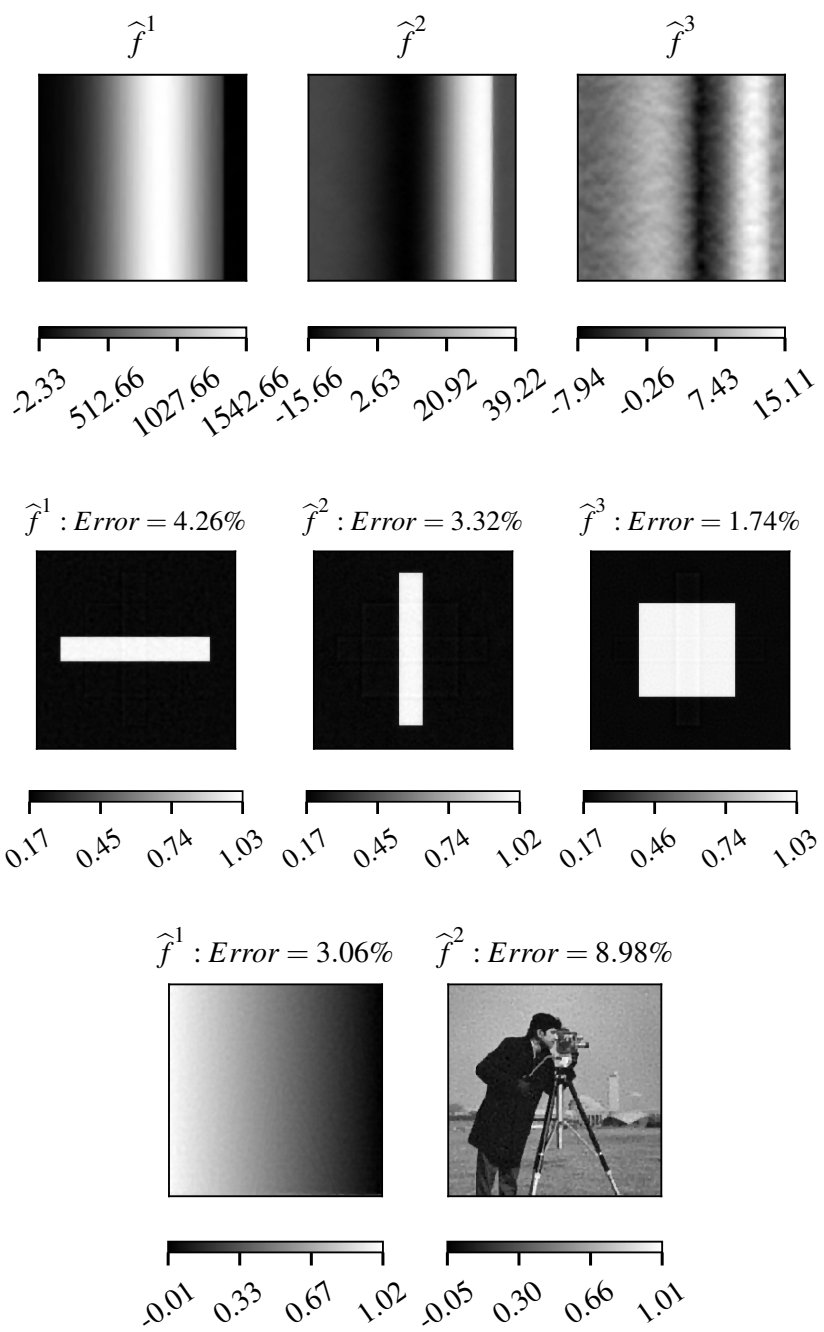

Fig. 6. Estimation results of the mixing coefficients associated to $\widehat{\mathrm{Obj}}_{1}$ (top), $\widehat{\mathrm{Obj}}_{2}$ (middle), and $\widehat{\mathrm{Obj}}_{3}$ (bottom). No error wrt. mixing coefficients is available for $\mathrm{Obj}_{1}$ since the original ones do not exist. 


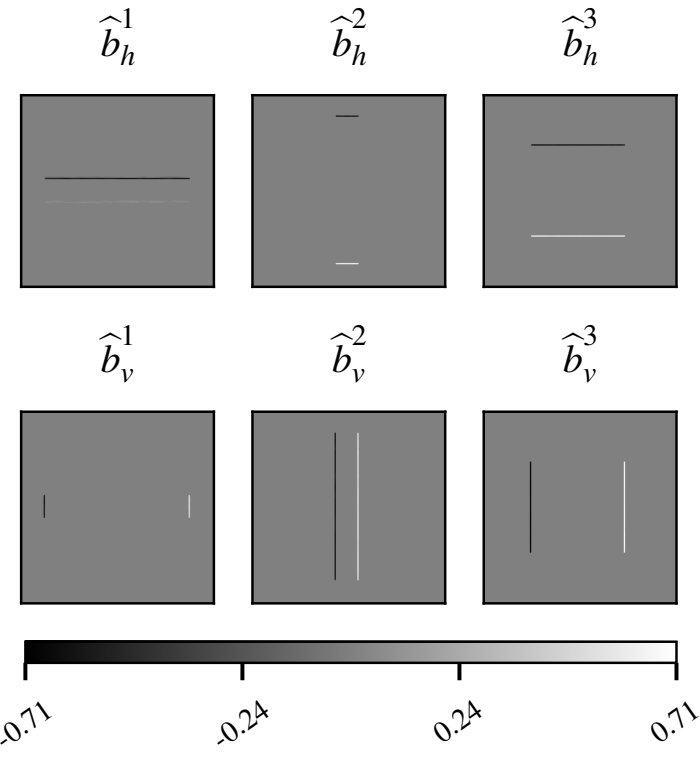

Fig. 7. Estimated auxiliary variables, horizontal and vertical, for $\widehat{\mathrm{Obj}}_{2}$.

Finally, the Fig. 12 shows the improvement of the spatial resolution at different wavelength values. Figures show the circular mean of the true Optical Transfer Function (OTF), that is the Fourier transform of the PSF, wrt. the "equivalent" OTF, which is the division between the true sky and the reconstructed sky in Fourier domain (for $\mathrm{Obj}_{3}$ at $30 \mathrm{~dB}$ ). The figure shows that in addition to the deconvolution effect and the high frequency restoration, the resolution is almost identical at all wavelength.

b) Spectral distribution: The spectral distributions for one spatial position are illustrated in Fig. 11, with a comparison between the spectrum of the original object and the reconstructed ones using the proposed and the TV algorithms. We see that the proposed algorithm produces spectra that correspond very closely to the original spectra at all wavelengths. This is due to:

- the linear mixing model with $M$ known spectral components which allow disentangling the spectral information integrated within each wideband image,

- the spectral variant PSF to model the instrument response accurately, hence the observation matrix $\boldsymbol{H}$,

- the spectral correlations between images exploited in joint reconstruction.

In contrast, the TV deconvolution method produces a poor spectral reconstruction since the spectral information within each band is not modeled (and implicitly flat).

\section{F. Influence of the Noise Level}

The reconstruction errors wrt. the noise levels are shown in Fig. 13. As expected, the proposed algorithm is sensitive to the noise with a decrease of the reconstruction errors for an increasing SNR. The reconstruction of $\mathrm{Obj}_{2}$ and $\mathrm{Obj}_{3}$ is very sensitive to the noise since the noise corrupts the sharp edges in the multispectral data, and make their restoration more

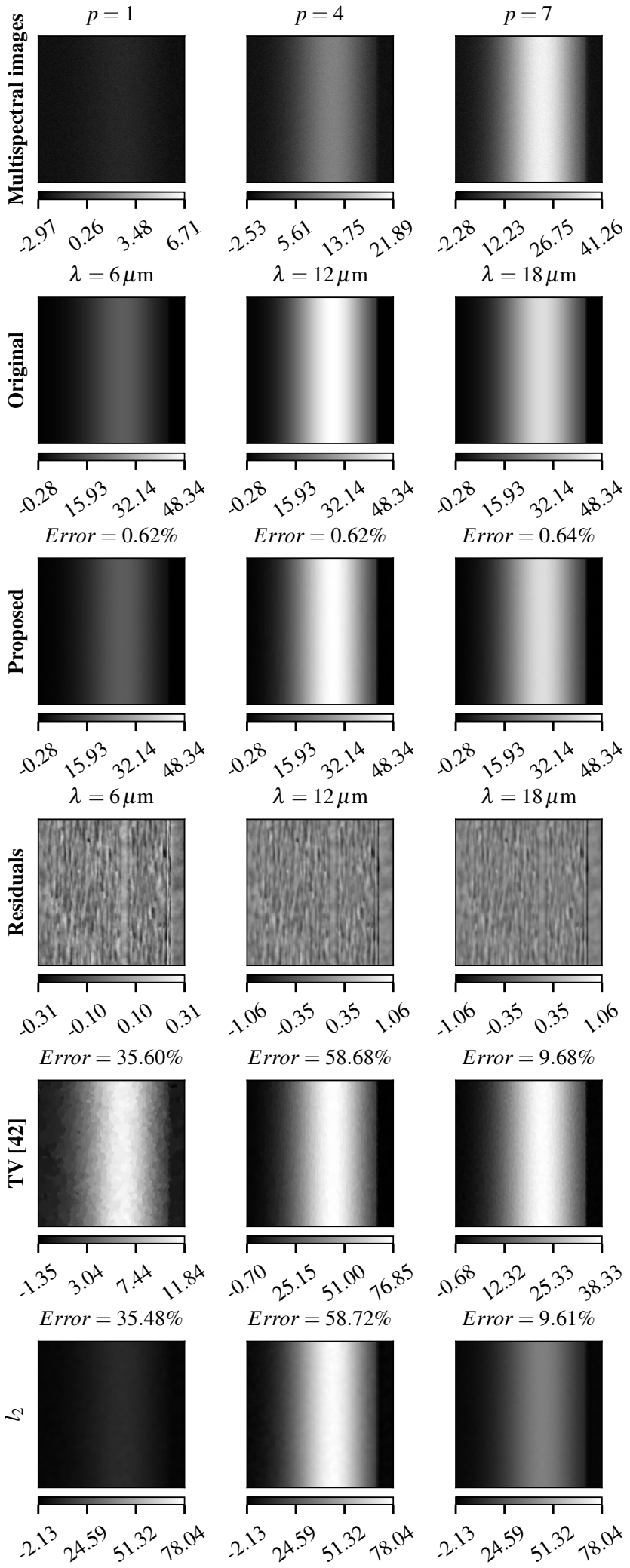

Fig. 8. Reconstruction for $\mathrm{Obj}_{1}$ at $30 \mathrm{~dB}$ : [1st row] Simulated multispectral images data at bands $p=1,4$, and 7 with a $\mathrm{SNR}=30 \mathrm{~dB}$. [2nd row] Original monochromatic images at 6,12 and $18 \mu \mathrm{m}$, contained in bands $p=$ 1, 4,7. [3rd row] Reconstruction at 6,12 and $18 \mu \mathrm{m}$. [4th row] Residuals. [5th row] Reconstruction with TV restoration. 
difficult. This is less the case for $\mathrm{Obj}_{1}$ which is dominated by a smoother spatial distribution.
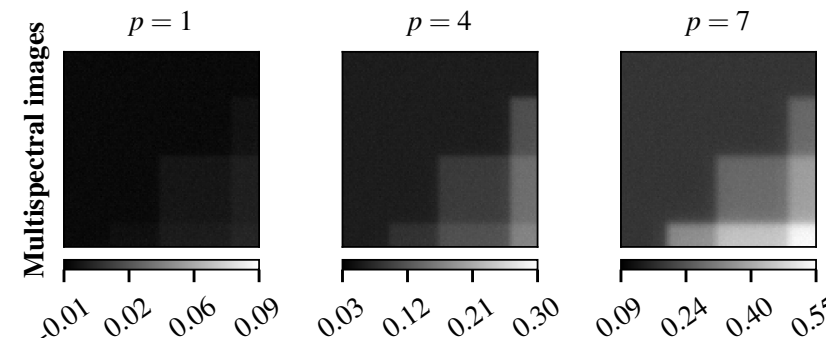

$\lambda=6 \mu \mathrm{m}$

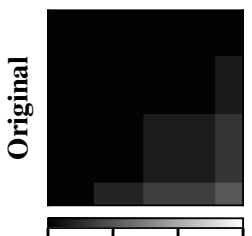

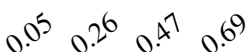

Error $=2.15 \%$

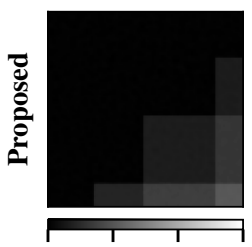

$0.050 .20 \quad 0.70 .0^{9}$

$\lambda=6 \mu \mathrm{m}$

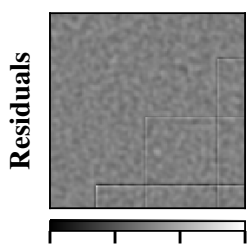

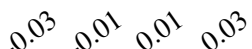

Error $=21.37 \%$

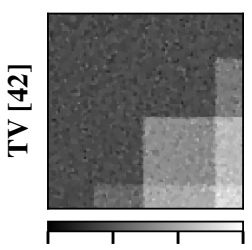

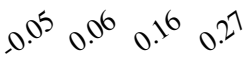

Error $=19.17 \%$

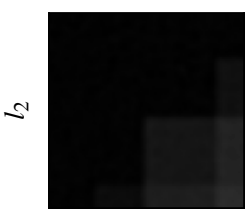

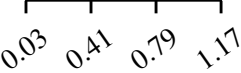

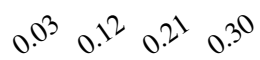

$0.9^{9} 0.2^{4} 0.4^{0} 0.5^{5}$

$$
\lambda=12 \mu \mathrm{m}
$$

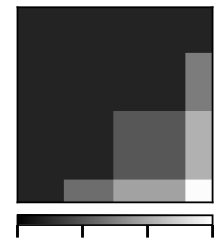

$0.050 .2^{6} \quad 0.40 .6^{.9}$

Error $=2.21 \%$

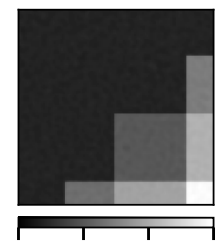

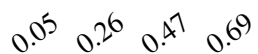

$\lambda=12 \mu \mathrm{m}$

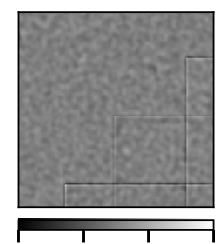

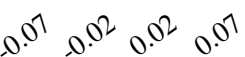

Error $=71.80 \%$

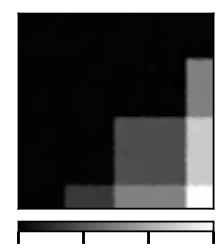

$0.210 .520 .820 . .^{3}$

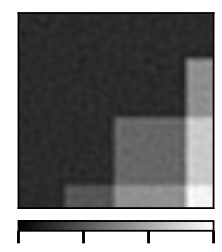

$0.0^{3} 0.4^{1 .} 0 . .^{9} \quad .1^{1}$ $\lambda=18 \mu \mathrm{m}$

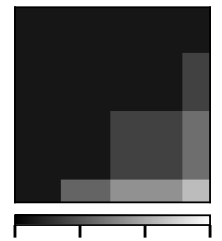

$0.0^{5} 0.2^{6} \quad 0 . x^{7} 0.6^{9}$

Error $=2.38 \%$

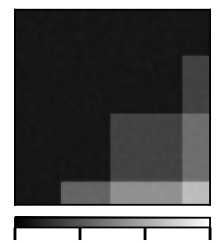

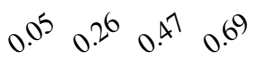

$\lambda=18 \mu \mathrm{m}$

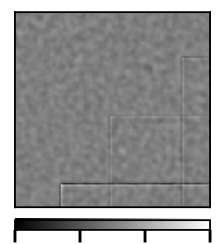

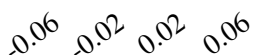

Error $=4.23 \%$

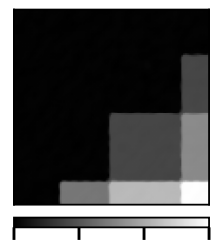

$0 . .^{0} 0.2^{4} 0.3^{9} 0.5^{33}$

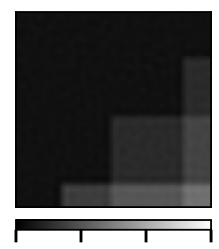

$0.0^{3} 0 . x^{.1} 0 . .^{9}, .17$
Error $=7.19 \%$
Error $=72.04 \%$

\section{CONCLUSION}

We present an efficient method for restoring from multispectral images the spectral and spatial information which are
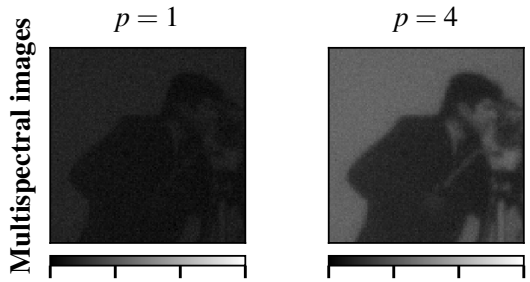

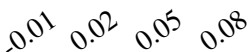
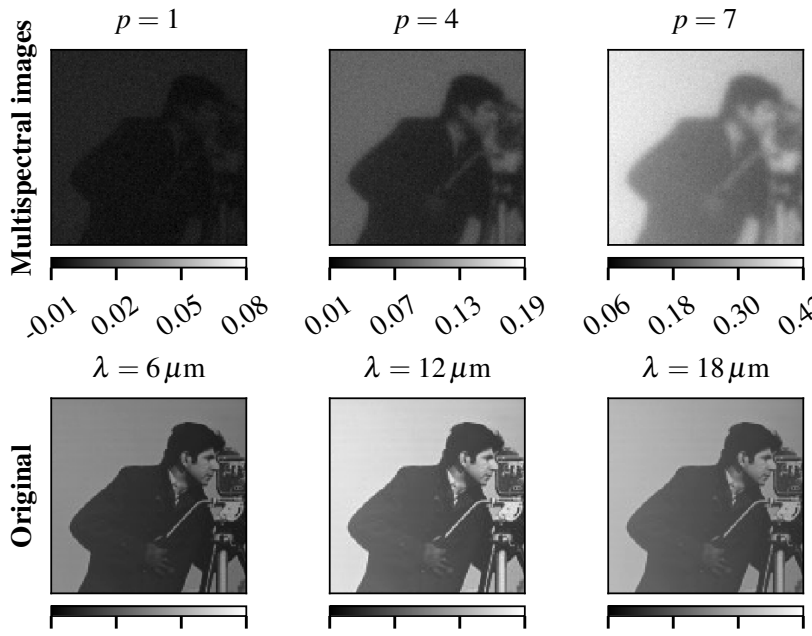

$\lambda=12 \mu \mathrm{m}$

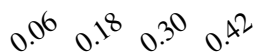
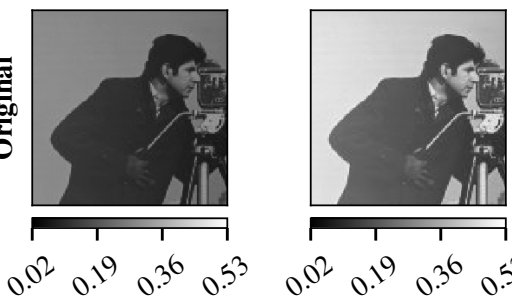

Error $=6.15 \%$

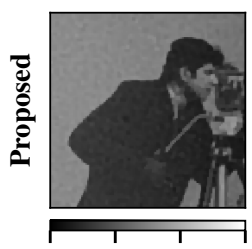

$0.0^{2} 0.1^{19} 0.3^{36} 0.5^{3}$

Error $=5.75 \%$

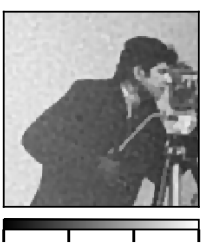

$0.0^{2} 0.9^{.9} 0.3^{30} 0.3^{3}$
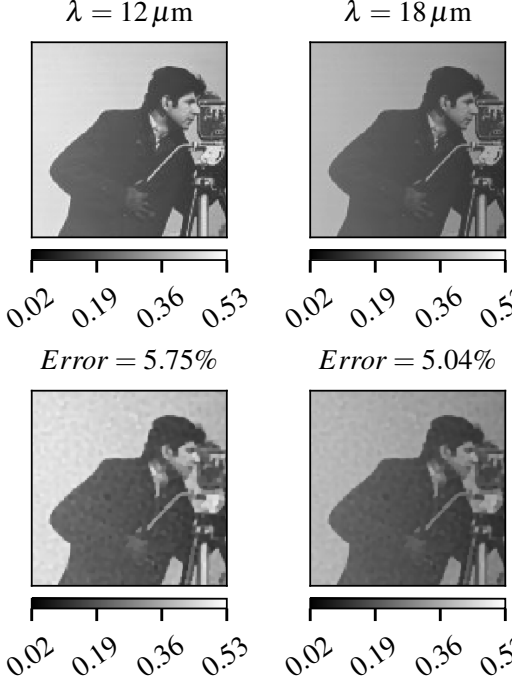

$0.0^{2} 0.9^{9} 0.3^{6} 0.5^{3}$

Error $=5.04 \%$
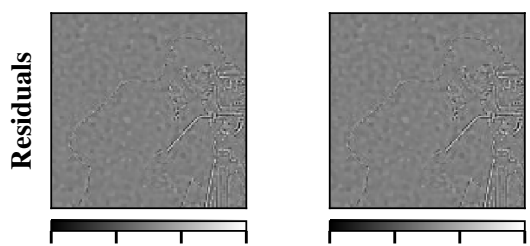

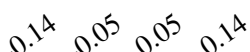
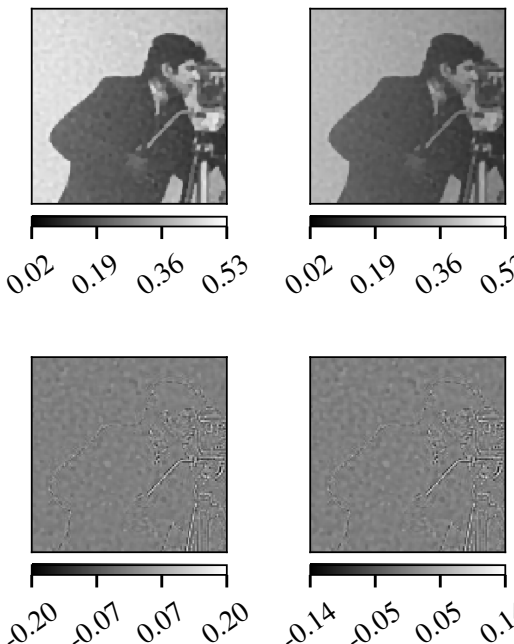

$0.0^{2} 0.9^{9} 0.3^{36} 0.5^{3}$

Error $=52.57 \%$

Error $=42.54 \%$
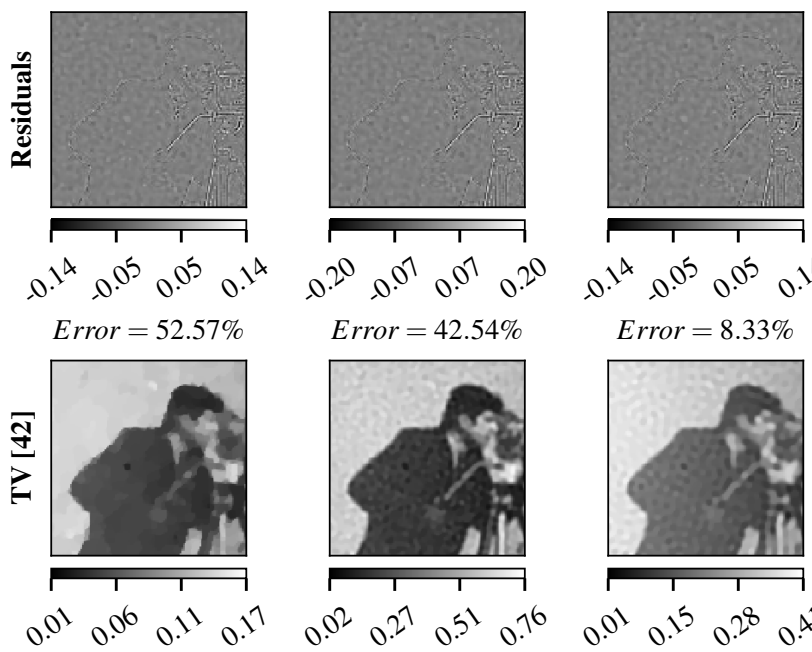

0.020 .210 .510 .16

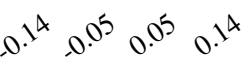

Error $=8.33 \%$

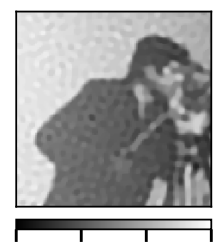

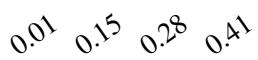

Error $=52.72 \%$

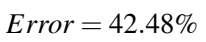

Error $=8.47 \%$
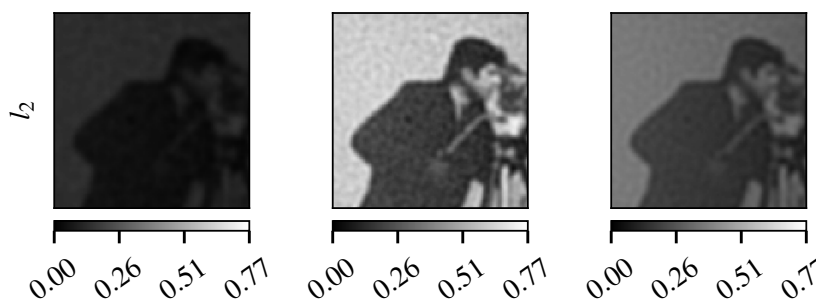

$0.0^{0} 0.2^{6} 0.5^{1} 0.7^{7}$ 
degraded in the acquisition process because of the spectral integration over wide bands, and the 2D convolution by the PSF (whose width linearly increases with wavelengths), which introduces a blur varying in space.

Our first contribution is a new data model that combines (1) a low rank subspace approximation of the fully resolved hyperspectral input object, and (2) a complete instrument model that takes into account the spectral variations of the PSF and the spectral response of the instrument. Then, a linear multi-observation forward model is derived, where data images appear as the sum of direct 2D convolutions of mixing coefficients allowing fast computation.

Our second contribution is a Fast Joint Multiband Reconstruction (FJMR) algorithm, an edge-preserving variational algorithm to process the full multispectral wideband images which are taken at a different spatial resolution. The proposed half-quadratic algorithm is iterative but we show that each sub-step is closed form and tractable with exact computation, especially for the quadratic step, even if the forward model is not stationary. Therefore, the algorithm is very fast with less than 4 seconds to process 9 images of size $256^{2}$ on a standard laptop.

The performance of the reconstruction algorithm is validated for three hyperspectral objects, including an astrophysical simulated object, having different spatial and spectral dis-
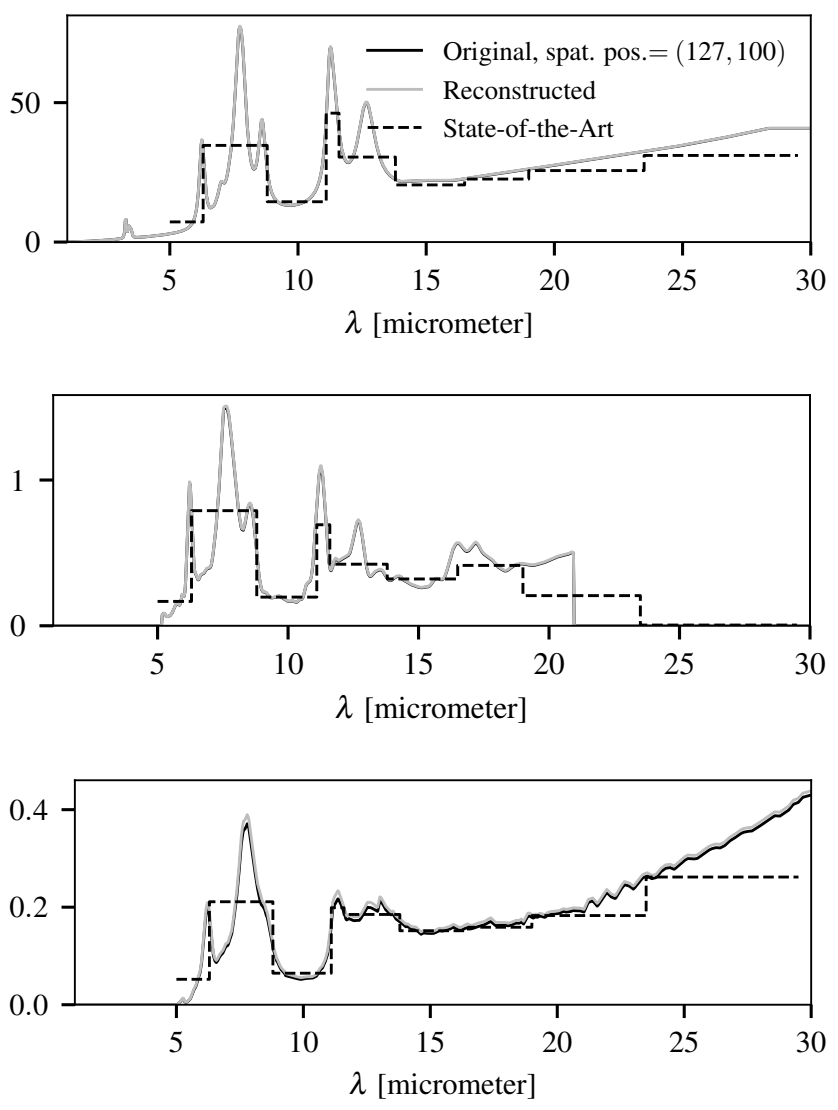

Fig. 11. Spectral distribution of the reconstruction results for one spatial position $(127,100)$ for $\mathrm{Obj}_{1}$ (top), $\mathrm{Obj}_{2}$ (middle) and $\mathrm{Obj}_{3}$ (bottom), with TV deconvolution as state-of-the-art method. The curves are in input sky unit.

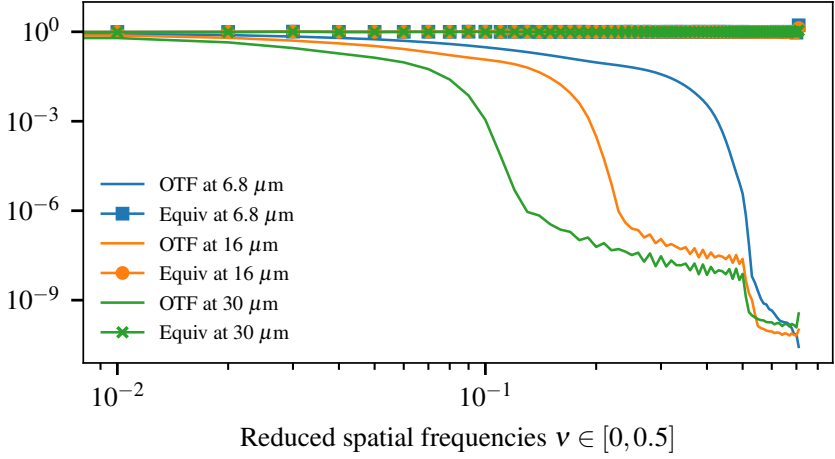

Fig. 12. Circular mean of the OTFs and the "equivalent" OTFs at three wavelengths, for $\mathrm{Obj}_{3}$ at $30 \mathrm{~dB}$. The "equivalent" OTF is the ratio between the true sky and the estimated sky at specified wavelengths. The three "equivalent" OTFs are superposed, indicating that the resolution is almost identical at these wavelengths.

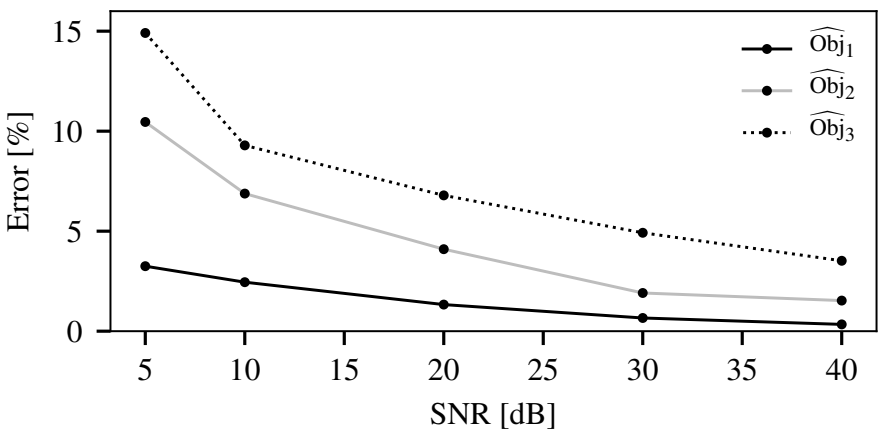

Fig. 13. Influence of the SNR on the reconstruction.

tributions that cover most of the encountered cases, in the context of the MIRI infrared imager of the JWST. In all experiments, the relative errors are below $5 \%$ for $\mathrm{SNR}=30$ $\mathrm{dB}$. In addition, the reconstruction results using the proposed algorithm significantly outperform the TV deconvolution. The sharp edges and small-scale gradients which are contained in the original objects but blurred in the multispectral images are correctly recovered. Thanks to our model where spatial resolution is defined by mixing coefficient only, the spatial resolution is homogenized at all wavelengths. Moreover, our algorithm allows us to recover the spectroscopic information contained within each band but lost in the data because of the spectral integration over the bands.

It is generally impossible to acquire spectroscopic data for large areas because the fields of view of spectrometers are generally very limited and much smaller than those of imagers. Moreover, the spectral coverage of observations becomes nowadays more and more extended. Therefore our algorithm, which has demonstrated its effectiveness to recover the spectroscopic information contained within wideband images and to reconstruct hyperspectral images with a homogenized spatial resolution at all wavelengths, appears a very promising tool that could be used for many scientific fields.

Many perspectives are possible. First the hyper parameters are fixed by hand, and their automatic estimation from data is a challenging question. Secondly, the spectral components 
are not always available and data with more spectral details must be used. Finally, the method supposes that all images are acquired with the same spatial sampling step, and without shifts between the images.

\section{APPENDIX A}

\section{COMPutation OF THE Multichannel QuAdRATiC SOLUTION}

In this section we present the computation of the multichannel quadratic solution given by

$$
\underbrace{\left[\begin{array}{c}
\widehat{\boldsymbol{f}}^{1} \\
\vdots \\
\widehat{\boldsymbol{f}}^{M}
\end{array}\right]}_{\widehat{\boldsymbol{f}}}=\underbrace{\left[\begin{array}{ccc}
\boldsymbol{Q}^{1,1} & \cdots & \boldsymbol{Q}^{1, M} \\
\vdots & \ddots & \vdots \\
\boldsymbol{Q}^{M, 1} & \cdots & \boldsymbol{Q}^{M, M}
\end{array}\right]}_{\boldsymbol{Q}} \underbrace{\left[\begin{array}{c}
\boldsymbol{q}^{1} \\
\vdots \\
\boldsymbol{q}^{M}
\end{array}\right]}_{\boldsymbol{q}},
$$

where $\boldsymbol{Q} \in \mathbb{R}^{M N_{k} N_{l} \times M N_{k} N_{l}}$ is a non-circulant block circulant matrix and $\boldsymbol{q} \in \mathbb{R}^{M N_{k} N_{l}}$ is a multichannel vector. The computation of $\widehat{f}$ relies on the inversion of the Hessian matrix $Q$ inspired from [7], [51]. Inverting $Q$ and storage of the inverse is possible by performing diagonalization of its circulant blocks $\boldsymbol{Q}^{i, j}$, resulting in a set of diagonal blocks $\boldsymbol{\Lambda}^{i, j}$ through the transfer equation

$$
\boldsymbol{Q}^{i, j}=\boldsymbol{F}^{\dagger} \boldsymbol{\Lambda}^{i, j} \boldsymbol{F}, \quad i, j \in[1, \ldots, M]^{2},
$$

where $\boldsymbol{F}$ and $\boldsymbol{F}^{\dagger}$ are the discrete Fourier matrix and its conjugate, respectively. This yields

$$
\boldsymbol{Q}=\overline{\boldsymbol{F}}^{\dagger} \boldsymbol{\Lambda}_{\boldsymbol{Q}} \overline{\boldsymbol{F}}, \quad \text { and } \quad \boldsymbol{Q}^{-1}=\overline{\boldsymbol{F}}^{\dagger} \boldsymbol{\Lambda}_{\boldsymbol{Q}}^{-1} \overline{\boldsymbol{F}},
$$

with

$$
\boldsymbol{\Lambda}_{\boldsymbol{Q}}=\left[\begin{array}{ccc}
\boldsymbol{\Lambda}^{1,1} & \cdots & \boldsymbol{\Lambda}^{1, M} \\
\vdots & \ddots & \vdots \\
\boldsymbol{\Lambda}^{M, 1} & \cdots & \boldsymbol{\Lambda}^{M, M}
\end{array}\right], \overline{\boldsymbol{F}}=\left[\begin{array}{ccc}
\boldsymbol{F} & & \\
& \ddots & \\
& & \boldsymbol{F}
\end{array}\right] .
$$

The matrix $\Lambda_{\boldsymbol{Q}}$ is a Non-Diagonal Block Diagonal (NDBD) matrix. Thanks to the permutation matrices $\boldsymbol{P}$, the NDBD matrix can be written as

$$
\Lambda_{Q}=\boldsymbol{P R P}
$$

where $\boldsymbol{R}=\operatorname{diag}\left(\boldsymbol{R}^{p}\right), p=1, \ldots, N_{k} N_{l}$ is a matrix with full blocks on the diagonal. Each block $\boldsymbol{R}^{p}$ is an $M \times M$ full matrix, invertible in our case, with permutations that writes

$$
\left(\boldsymbol{R}^{p}\right)_{i, j}=\left(\boldsymbol{\Lambda}^{i, j}\right)_{p, p}
$$

for $i, j \in[1, \ldots, M]^{2}$. Therefore, since the inversion of a block diagonal matrix is also a block diagonal matrix, the inverse of $\Lambda_{Q}$ can be written as

$$
\Upsilon:=\Lambda_{\boldsymbol{Q}}^{-1}=\boldsymbol{P}^{T} \boldsymbol{R}^{-1} \boldsymbol{P}^{T}
$$

where $\boldsymbol{R}^{-1}=\operatorname{diag}\left(\left(\boldsymbol{R}^{p}\right)^{-1}\right)$, and $\boldsymbol{\Upsilon}$ is also a NDBD matrix, having a diagonal block $\Upsilon^{i, j}$ given by

$$
\left(\boldsymbol{\Upsilon}^{i, j}\right)_{p, p}=\left(\left(\boldsymbol{R}^{p}\right)^{-1}\right)_{i, j} .
$$

In conclusion, $\Upsilon$ is tractable and sparse, allowing pre computation and direct application in the iterative Algorithm 1.
Thanks to such properties, the multichannel quadratic solution can be computed with the DFT and $N_{k} N_{l}$ inversions of square matrices of size $M$. In the context of this work, $N_{k} N_{l}$ are the number of pixels and $M$ is the number of spectral components. In addition, each block $\boldsymbol{R}^{p}$ can be inverted in parallel. Finally, $\widehat{f}$ is computed just by applying the matrix $\Upsilon$ as

$$
\widehat{\boldsymbol{f}}=\overline{\boldsymbol{F}}^{\dagger} \boldsymbol{\Lambda}_{\boldsymbol{Q}}^{-1} \stackrel{\circ}{\boldsymbol{q}}=\overline{\boldsymbol{F}}^{\dagger} \boldsymbol{\Upsilon} \overline{\boldsymbol{F}} \boldsymbol{q}
$$

with $\stackrel{\stackrel{q}{ }=}{=} \overline{\boldsymbol{F}} \boldsymbol{q}$.

\section{APPENDIX B}

\section{UPDATE OF THE AUXILIARY VARIABLES}

The update of all auxiliary variables is independent and can be calculated in parallel. The solution is given by the minimization

$$
\widehat{\boldsymbol{b}}^{m}=\underset{\boldsymbol{b}^{m}}{\operatorname{argmin}} \underbrace{\frac{1}{2}\left(\left[\boldsymbol{D} \boldsymbol{f}^{m}\right]_{k, l}-\left[\boldsymbol{b}^{m}\right]_{k, l}\right)^{2}+\xi\left(\left[\boldsymbol{b}^{m}\right]_{k, l}\right)}_{\psi\left(\left[\boldsymbol{b}^{m}\right]_{k, l}\right)}
$$

where $\psi$ is a convex, differentiable, and separable function with respect to the minimizer. For the Huber potential $\varphi$, the auxiliary function is $\xi(b)=2 s|b|$. Since the criterion is convex differentiable, a sufficient condition is $\psi^{\prime}\left(\left[\widehat{\boldsymbol{b}}^{m}\right]_{k, l}\right)=0$, $\forall k \in\left[1, N_{k}\right]$ and $\forall l \in\left[1, N_{l}\right]$. The derivative function $\psi^{\prime}$ is computed by substituting the auxiliary function in $\psi$. This yields for the Huber potential

$\psi^{\prime}\left(\left[\widehat{\boldsymbol{b}}^{m}\right]_{k, l}\right)=\left(\left[\widehat{\boldsymbol{b}}^{m}\right]_{k, l}-\left[\boldsymbol{D} \boldsymbol{f}^{m}\right]_{k, l}\right)+s \operatorname{sign}\left(\left[\widehat{\boldsymbol{b}}^{m}\right]_{k, l}\right)$.

Thus, the obtained auxiliary variables are

$$
\begin{aligned}
{\left[\widehat{\boldsymbol{b}}^{m}\right]_{k, l} } & =\left\{\begin{array}{l}
{\left[\boldsymbol{D} \boldsymbol{f}^{m}\right]_{k, l}-\left[\boldsymbol{D} \boldsymbol{f}^{m}\right]_{k, l}, \text { if }\left|\left[\boldsymbol{D} \boldsymbol{f}^{m}\right]_{k, l}\right|<s} \\
{\left[\boldsymbol{D} \boldsymbol{f}^{m}\right]_{k, l}-s \operatorname{sign}\left(\left[\boldsymbol{D} \boldsymbol{f}^{m}\right]_{k, l}\right), \text { otherwise. }}
\end{array}\right. \\
& =\left[\boldsymbol{D} \boldsymbol{f}^{m}\right]_{k, l}-\frac{1}{2} \varphi^{\prime}\left(\left[\boldsymbol{D} \boldsymbol{f}^{m}\right]_{k, l}\right) .
\end{aligned}
$$

Finally, we obtain

$$
\widehat{\boldsymbol{b}}=\boldsymbol{D} \boldsymbol{f}-\frac{1}{2} \varphi^{\prime}(\overline{\boldsymbol{D}} \boldsymbol{f})
$$

where $\varphi^{\prime}$ is the first derivative of Huber function

$$
\varphi^{\prime}(x)= \begin{cases}2 x, & \text { if }|x|<s, \\ 2 s \operatorname{sign}(x), & \text { otherwise. }\end{cases}
$$

\section{ACKNOWLEDGMENT}

The authors would like to thank Nathalie Ysard for kindly providing the simulation of the hyperspectral object HorseHead.

\section{REFERENCES}

[1] R. Lionello, J. A. Linker, and Z. Mikić, "Multispectral emission of the sun during the first whole sun month: Magnetohydrodynamic simulations," The Astrophysical Journal, vol. 690, no. 1, p. 902, 2008.

[2] D. A. Landgrebe, Signal theory methods in multispectral remote sensing. John Wiley \& Sons, 2005, vol. 29. 
[3] T. O. McBride, B. W. Pogue, S. Poplack, S. Soho, W. A. Wells, S. Jiang, K. D. Paulsen et al., "Multispectral near-infrared tomography: a case study in compensating for water and lipid content in hemoglobin imaging of the breast," Journal of biomedical optics, vol. 7, no. 1, pp. 72-79, 2002.

[4] M. Dickinson, G. Bearman, S. Tille, R. Lansford, and S. Fraser, "Multispectral imaging and linear unmixing add a whole new dimension to laser scanning fluorescence microscopy," Biotechniques, vol. 31, no. 6 , pp. 1272-1279, 2001.

[5] J. W. Goodman, Introduction to Fourier optics. Roberts and Company Publishers, 2005.

[6] P. Guillard, T. Rodet, S. Ronayette, J. Amiaux, A. Abergel, V. Moreau, J. Augueres, A. Bensalem, T. Orduna, C. Nehmé et al., "Optical performance of the jwst/miri flight model: characterization of the point spread function at high resolution," in SPIE Astronomical Telescopes + Instrumentation. International Society for Optics and Photonics, 2010, pp. $77310 \mathrm{~J}-77310 \mathrm{~J}$.

[7] N. P. Galatsanos and R. T. Chin, "Digital restoration of multichannel images," IEEE Transactions on Acoustics, Speech, and Signal Processing, vol. 37, no. 3, pp. 415-421, 1989.

[8] L. Denis, É. Thiébaut, and F. Soulez, "Fast model of space-variant blurring and its application to deconvolution in astronomy," in Image Processing (ICIP), 2011 18th IEEE International Conference on. IEEE, 2011, pp. $2817-2820$

[9] É. Thiébaut, L. Dénis, F. Soulez, and R. Mourya, "Spatially variant psf modeling and image deblurring," in SPIE Astronomical Telescopes+ Instrumentation. International Society for Optics and Photonics, 2016, pp. $99097 \mathrm{~N}-99097 \mathrm{~N}$.

[10] G. Aniano, B. Draine, K. Gordon, and K. Sandstrom, "Commonresolution convolution kernels for space-and ground-based telescopes," Publications of the Astronomical Society of the Pacific, vol. 123, no. 908, p. 1218, 2011.

[11] A. Boucaud, M. Bocchio, A. Abergel, F. Orieux, H. Dole, and M. A. Hadj-Youcef, "Convolution kernels for multi-wavelength imaging," Astronomy \& Astrophysics, vol. 596, p. A63, 2016.

[12] N. Keshava and J. F. Mustard, "Spectral unmixing," IEEE signal processing magazine, vol. 19, no. 1, pp. 44-57, 2002.

[13] A. Plaza, P. Martínez, R. Pérez, and J. Plaza, "A quantitative and comparative analysis of endmember extraction algorithms from hyperspectral data," IEEE transactions on geoscience and remote sensing, vol. 42, no. 3, pp. 650-663, 2004.

[14] J. M. Bioucas-Dias, A. Plaza, N. Dobigeon, M. Parente, Q. Du, P. Gader, and J. Chanussot, "Hyperspectral unmixing overview: Geometrical, statistical, and sparse regression-based approaches," IEEE journal of selected topics in applied earth observations and remote sensing, vol. 5, no. 2, pp. 354-379, 2012

[15] J. B. Adams, M. O. Smith, and P. E. Johnson, "Spectral mixture modeling: A new analysis of rock and soil types at the viking lander 1 site," Journal of Geophysical Research: Solid Earth, vol. 91, no. B8, pp. 8098-8112, 1986.

[16] J. Settle and N. Drake, "Linear mixing and the estimation of ground cover proportions," International Journal of Remote Sensing, vol. 14, no. 6, pp. 1159-1177, 1993.

[17] V. Haertel and Y. E. Shimabukuro, "Spectral linear mixing model in low spatial resolution image data," in Geoscience and Remote Sensing Symposium, 2004. IGARSS'04. Proceedings. 2004 IEEE International, vol. 4. IEEE, 2004, pp. 2546-2549.

[18] O. Berne, C. Joblin, Y. Deville, J. Smith, M. Rapacioli, J. Bernard, J. Thomas, W. Reach, and A. Abergel, "Analysis of the emission of very small dust particles from spitzer spectro-imagery data using blind signal separation methods," Astronomy \& Astrophysics, vol. 469, no. 2, pp. 575-586, 2007.

[19] N. Dobigeon, S. Moussaoui, M. Coulon, J.-Y. Tourneret, and A. O. Hero, "Joint bayesian endmember extraction and linear unmixing for hyperspectral imagery," IEEE Transactions on Signal Processing, vol. 57, no. 11, pp. 4355-4368, 2009.

[20] Y. Tarabalka, J. A. Benediktsson, and J. Chanussot, "Spectral-spatial classification of hyperspectral imagery based on partitional clustering techniques," IEEE Transactions on Geoscience and Remote Sensing, vol. 47, no. 8, pp. 2973-2987, 2009.

[21] Z. Guo, T. Wittman, and S. Osher, "L1 unmixing and its application to hyperspectral image enhancement," in Algorithms and Technologies for Multispectral, Hyperspectral, and Ultraspectral Imagery XV, vol. 7334. International Society for Optics and Photonics, 2009, p. 73341M.

[22] L. Loncan, L. B. Almeida, J. M. Bioucas-Dias, X. Briottet, J. Chanussot, N. Dobigeon, S. Fabre, W. Liao, G. A. Licciardi, M. Simoes et al., "Hy- perspectral pansharpening: A review," arXiv preprint arXiv:1504.04531, 2015.

[23] F. Orieux, J.-F. Giovannelli, and T. Rodet, "Bayesian estimation of regularization and point spread function parameters for wiener-hunt deconvolution," JOSA A, vol. 27, no. 7, pp. 1593-1607, 2010.

[24] J. Yang, Y. Zhang, and W. Yin, "An efficient tvll algorithm for deblurring multichannel images corrupted by impulsive noise," SIAM Journal on Scientific Computing, vol. 31, no. 4, pp. 2842-2865, 2009.

[25] A. Chambolle and T. Pock, "A first-order primal-dual algorithm for convex problems with applications to imaging," Journal of Mathematical Imaging and Vision, vol. 40, no. 1, pp. 120-145, 2011.

[26] P. Bouchet, M. García-Marín, P.-O. Lagage, J. Amiaux, J.-L. Auguéres, E. Bauwens, J. Blommaert, C. Chen, Ö. Detre, D. Dicken et al., "The Mid-Infrared Instrument for the James Webb Space Telescope, III MIRIM, The MIRI Imager," Publications of the Astronomical Society of the Pacific, vol. 127, no. 953, p. 612, 2015.

[27] J. P. Gardner, J. C. Mather, M. Clampin, R. Doyon, M. A. Greenhouse, H. B. Hammel, J. B. Hutchings, P. Jakobsen, S. J. Lilly, K. S. Long et al., "The james webb space telescope," Space Science Reviews, vol. 123, no. 4, pp. 485-606, 2006.

[28] M. A. Hadj-Youcef, F. Orieux, A. Fraysse, and A. Abergel, "Spatiospectral multichannel reconstruction from few low-resolution multispectral data," in 2018 26th European Signal Processing Conference (EUSIPCO), Sep 2018.

[29] I. T. Jolliffe, "Principal component analysis and factor analysis," in Principal component analysis. Springer, 1986, pp. 115-128.

[30] D. D. Lee and H. S. Seung, "Algorithms for non-negative matrix factorization," in Advances in neural information processing systems, 2001, pp. 556-562.

[31] S. Bongard, F. Soulez, É. Thiébaut, and É. Pecontal, "3d deconvolution of hyper-spectral astronomical data," Monthly Notices of the Royal Astronomical Society, vol. 418, no. 1, pp. 258-270, 2011.

[32] B. Hunt, "A matrix theory proof of the discrete convolution theorem," IEEE Transactions on Audio and Electroacoustics, vol. 19, no. 4, pp. 285-288, 1971.

[33] G. Demoment, "Image reconstruction and restoration: Overview of common estimation structures and problems," IEEE Transactions on Acoustics, Speech, and Signal Processing, vol. 37, no. 12, pp. 20242036, 1989.

[34] A. N. Tikhonov, A. Goncharsky, V. Stepanov, and A. G. Yagola, Numerical methods for the solution of ill-posed problems. Springer Science \& Business Media, 2013, vol. 328.

[35] L. I. Rudin, S. Osher, and E. Fatemi, "Nonlinear total variation based noise removal algorithms," Physica D: Nonlinear Phenomena, vol. 60, no. 1, pp. 259-268, 1992

[36] I. Daubechies and G. Teschke, "Wavelet based image decomposition by variational functionals," in Proc. SPIE Vol, vol. 5266, 2004, pp. 94-105.

[37] M. A. Figueiredo and R. D. Nowak, "An em algorithm for wavelet-based image restoration," IEEE Transactions on Image Processing, vol. 12, no. 8, pp. 906-916, 2003.

[38] D. Geman and G. Reynolds, "Constrained restoration and the recovery of discontinuities," IEEE Transactions on pattern analysis and machine intelligence, vol. 14, no. 3, pp. 367-383, 1992.

[39] D. Geman and C. Yang, "Nonlinear image recovery with half-quadratic regularization," IEEE Transactions on Image Processing, vol. 4, no. 7, pp. 932-946, 1995.

[40] P. Perona and J. Malik, "Scale-space and edge detection using anisotropic diffusion," IEEE Transactions on pattern analysis and machine intelligence, vol. 12, no. 7, pp. 629-639, 1990.

[41] A. Chambolle, "An algorithm for total variation minimization and applications," Journal of Mathematical imaging and vision, vol. 20, no. 1-2, pp. 89-97, 2004.

[42] P. Charbonnier, L. Blanc-Féraud, G. Aubert, and M. Barlaud, "Deterministic edge-preserving regularization in computed imaging," IEEE Transactions on image processing, vol. 6, no. 2, pp. 298-311, 1997.

[43] E. Chouzenoux, A. Jezierska, J.-C. Pesquet, and H. Talbot, "A majorizeminimize subspace approach for $\ell_{2}-\ell_{0}$ image regularization," SIAM Journal on Imaging Sciences, vol. 6, no. 1, pp. 563-591, 2013.

[44] J. Idier, "Convex half-quadratic criteria and interacting auxiliary variables for image restoration," IEEE Transactions on Image Processing, vol. 10, no. 7, pp. 1001-1009, 2001.

[45] M. D. Perrin, R. Soummer, E. M. Elliott, M. D. Lallo, and A. Sivaramakrishnan, "Simulating point spread functions for the james webb space telescope with webbpsf," in Space Telescopes and Instrumentation 2012: Optical, Infrared, and Millimeter Wave, vol. 8442. International Society for Optics and Photonics, 2012, p. 84423D. 
[46] M. D. Perrin, A. Sivaramakrishnan, C.-P. Lajoie, E. Elliott, L. Pueyo, S. Ravindranath, and L. Albert, "Updated point spread function simulations for jwst with webbpsf," in Space Telescopes and Instrumentation 2014: Optical, Infrared, and Millimeter Wave, vol. 9143. International Society for Optics and Photonics, 2014, p. 91433X.

[47] A. Glasse, G. Rieke, E. Bauwens, M. García-Marín, M. Ressler, S. Rost, T. V. Tikkanen, B. Vandenbussche, and G. Wright, "The Mid-Infrared Instrument for the James Webb Space Telescope, IX: Predicted Sensitivity," Publications of the Astronomical Society of the Pacific, vol. 127, no. 953 , p. 686,2015

[48] U. of Arizona, "Table of integration window for each filter," 2018, accessed 2018-31-10. [Online]. Available: http://ircamera.as.arizona. edu/MIRI/ImPCE_TN-00072-ATC-Iss2.xlsx

[49] A. Abergel, D. Teyssier, J. Bernard, F. Boulanger, A. Coulais, D. Fosse, E. Falgarone, M. Gerin, M. Perault, J.-L. Puget et al., "Isocam and molecular observations of the edge of the horsehead nebula," Astronomy \& Astrophysics, vol. 410, no. 2, pp. 577-585, 2003.

[50] J. R. Houck, T. L. Roellig, van Cleve, and et al., "The Infrared Spectrograph (IRS) on the Spitzer Space Telescope," Astrophysical Journal Supplement Series, vol. 154, no. 1, pp. 18-24, Sep 2004.

[51] N. P. Galatsanos, M. N. Wernick, A. K. Katsaggelos, and R. Molina, "Multichannel image recovery," Handbook of Image and Video Processing, vol. 12, pp. 155-168, 2000.

M. Amine Hadj-Youcef was born in Blida, Algeria, in 1989. He holds a MSc degree and a Ph.D from the University of Bordeaux, France, and the University of Paris-Saclay, France, in 2015 and 2018, respectively.

Since then, he is working in Research and development for industry, in various fields such as artificial intelligence based computer vision, machine learning, and data science. He recently joined the R\&D department at TAG Heuer Connected, Paris, France.

Francois Orieux is Assistant Professor at the Université Paris-Sud and a researcher in the Laboratoire des Signaux et Systèmes, Groupe Problèmes Inverses (Université Paris-Saclay, CNRS, CentraleSupélec), France. He is also an associate researcher with the Institut d'Astrophysique Spatiale (Univ. ParisSaclay, CNRS).

He received his Ph.D. degree in signal processing at Université Paris-Sud, Orsay, France. His research focuses on Bayesian methodological approaches for ill-posed inverse problems resolution with examples of applications in astrophysics or biological microscopy.

Alain Abergel is professor of Physics and Astrophysics at the Paris-Saclay University. He is astrophysicist of the interstellar medium at the Institut d'Astrophysique Spatiale (Paris-Saclay University, CNRS), and is involved in the scientific exploitation of several space missions for astrophysics (ISO, Spitzer, Herschel, Planck, JWST, ...).

Aurélia Fraysse was born in Creteil, France, in 1978. She graduated from University Paris 12 in 2001. She received her Ph. D. degree in mathematics at University Paris 12 in 2005.

In 2006, she was a research assistant at Ecole Nationale Supérieure des Télécommunications (Telecom Paris). She is presently assistant professor at IUT Cachan and researcher with the Laboratoire des Signaux et Systèmes (CNRS-CentraleSupelec-Univ. Paris-Sud). 